\title{
SUPERDAN: Coinpiter Programs for Calculating the Dancoff Factor of Spheres, Cylinders, and Slabs
}

\author{
J. R. Knight
}

Prepared for the U.S. Nuclear Regulatory Commission Office of Nuclear Regulatory Research Under Interagency Agreement DOE 40-550-75 
ORNL/NUREG/CSD/TM-2

Distribution Category

MRC-14

Contract No. H-7405 eng 26

CORPITER SCIENCES DIYISION

SUPERDAN: Computer Programs for cialculating the Dancoff

Factor of Spheres, Cylinders, and Slabs

J. R. Knight

Date Pub11shed - March 1978

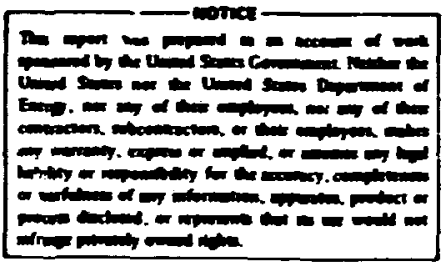

NOTICE This document contains information of preliminary nature. It is subjer,t to revision or correction and therefore does not represent a final report.

Prepared for the U.S. Nuclear Regulatory Commission

Office of Nuclear Regulatory Research

Under Interagency Agreement DOE 40-550-75

UNION CARBIDE CORFORATION, NUCLEAR DIVISION operating the

Oak Ridge Gaseous Diffusion Plant . Oak Ridge National Laboratory Oak Ridge $Y-12$ Plant - Paducah Gaseous Diffusion Plant for the DEPARTMENT OF ENERGY 
TABLE OF CONTENTS

Page

ABSTRACT ............................. vii

IRTROCUCTION ............................ ix

I. CYLIRDRICAL LATTICES .................. 1

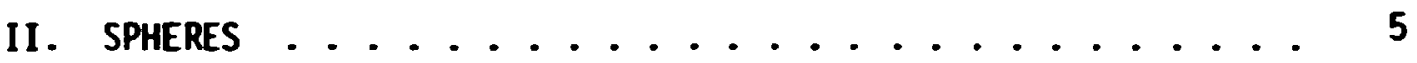

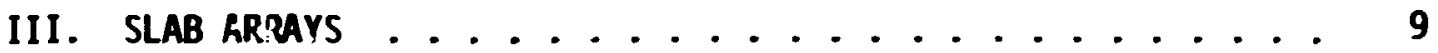

IV. RESULTS .......................... 11

TABLE 1. DANCOFF FACTORS FOR SPHERES .......... 12

ACKHOMLEDGHENTS ........................ 17

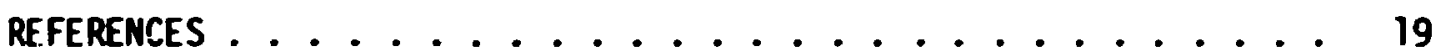

APPENDIX A. CYLINDER DANCOFF LIMITS OF INTEGRATION ...... 23

APPENDIX B. CHANGES IN THE LIMITS OF IHTEGRATION FOR

SQUARE PITCH ARRAYS OF CYLINDERS DUE TO SHADOWIMG EFFFCTS . . 27

APPENDIX C. CHANGES IN THE LIMITS OF INTEGRATION FOR TRI-

ANGULAR PITCH ARRAYS OF CYLINDERS DUE TO SHADONING EFFECTS . . . 37

APPENDIX D. THE EFFECT OF CLAD ON THE DANCOFF FACTOR IN

CYLIMDRICAL GEOMETRY .................... 49

APPENDIX E. DERIVATION OF THE EXPRESSION FOR COS $\alpha$ FOR THE

SPHERICAL DANCOFF FACTOR ............. 55

APPENDIX $F$. METHOD FOR CALCILATING VALUES OF THE $E_{3}(x)$

FUNCTION $\cdots$ 


\section{LIST CF FIGURES}

FIGURE

PAGE

1. Limits of integration ................ 2

2. Elements of surface area .............. 6

A1. Limiting angle $\theta_{\mathrm{L}}$................. 23

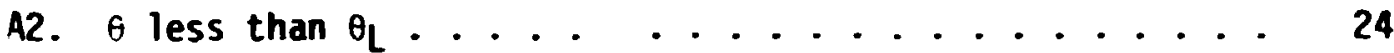

A3. A greater than $\theta_{\mathrm{L}} \ldots . . . . . . . . . . . . .25$

B1. Square array .................... 27

B2. Limiting spacing for shacowing of $\theta_{2}^{\prime} \ldots \ldots$........ 28

B3. Limiting spacing for $\theta_{i}$ shadowing . . . . . . . . . 29

B4. Limiting values of $\theta$ for $\theta_{2}^{\prime}$ shadowing .......... 31

B5. Limiting value of $\theta$ for $\jmath_{1}^{\prime}$ shadowing .......... 32

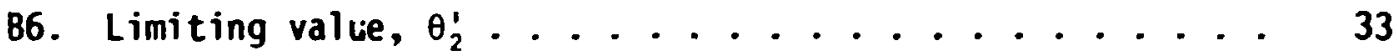

Cl. Triangular array of cylinders ........... 37

C2. Limiting spacing for shidowing of $\theta_{2}^{\prime} \ldots \ldots 38$

C3. Limiting values of $\theta \mathrm{fcr} \mathrm{g}_{2}^{\prime}$ shadowing ......... 39

C4. Limiting value, $\theta_{2}^{\prime} \ldots \ldots . . . . . . . . . .40$

C5. Limiting spacing for $\theta$ : shadowing ........... 41

C6. Limiting spacing for $\varsigma^{\text {: }}$ shadowing . . . . . . . . . 42

C7. Limiting values of $\theta$ fór $A_{2}^{\prime}$ shadowing . . . . . . . . 44

C8. Limiting value of $\theta$ for $\theta_{1}^{\prime}$ shadowing .......... 45

C9. Limiting value, $9_{2}^{\prime}$. . . . . . . . . . . . . 46

D1. Cylinder with clad ............... 50

E1. Cos a for two spheres .............. 56 


\begin{abstract}
Resonance integral calculations for a reactor lattice require the use of the Dancoff factor. In the past, tables and approximate expressions have been used to obtain tris value. In order to reduce the uncertainty in the use of approy :mations and to avoid the inconvenience of tables, a series of compuier prograns has been written to calculate the Dancoff factor.

Three computer programs have been prepared, one each for infinitely long cylinders, spheres, and infinite slabs. The program for cylincers includes corrections for the effect of partial shadowing of a rod by adjaceat rods and for the effect of clad surrounding the rods. The effect of clad is also considered in the slab program. Although these corrections can be applied to sphere arrays, they have not been inclused in the sphere program. It was felt that the infrequent use of spherical arrays jid not justify the additional time and effort to include these corrections.
\end{abstract}


In a lattice of rods, the presence of nearby units reduces the flux at a given resonance energy wich is incident on a given rod. The Cancoff: factor is used to determine this flux reduction in the resonance integral calculations. In the past, tables of the Dancoff factor published in ANL $-5800^{2}$ have been used extensively. In addition, several approximate expressions for the Danceff factor, such as the one given by Sauer, ${ }^{3}$ have been used. In order to reduce the uncertainty in the use of approximations and to avoid the inconvenience of tables, a series of computer prograns has been written to calculate the Dancoff factor.

Three computer programs have been prepared, one each for infinitely long cylinders, spheres, and infinite slabs. The program for cylinders includes corrections for the effect of partial shadowing of a rod by adjacent rods and for the effect of clad surrounding the rods. The effect of clad is also considered in the slab program. Although these corrections can be applied to sphere arrays, they have nct been included in the spnere program. It was felt that the infrequent use of spherical arrays did not justify the additional time and effort to include these corrections. 


\section{CYLINDRICAL LATTICES}

For the case of two cylindrical rods, Dancoff and Ginsburg ${ }^{1}$ give the expression:

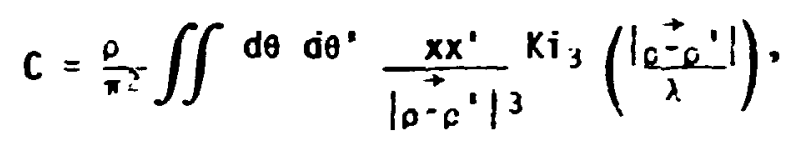

where:

$$
\begin{aligned}
& C=\text { Dancoff facter, } \\
& \rho=\text { rod radius, } \\
& \theta=\text { azimuth angle in cylinder } i \text {, } \\
& \theta^{\prime}=\text { azimuth angle in cylinder 2, } \\
& x=d \cos \theta-\rho-\rho \cos \left(\theta+g^{\prime}\right) \star \text {. } \\
& x^{\prime}=d \cos \theta^{\prime}-\rho-\rho \cos \left(\theta+\theta^{\circ}\right) \text {, }
\end{aligned}
$$

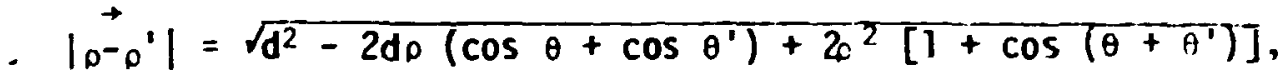

$$
\begin{aligned}
& d=\text { pitch, } \\
& \lambda=\text { moderator mean free path, } \\
& \mathrm{Ki}_{3}(\mathrm{x})=\text { Bickley function }{ }^{4} \text {. }
\end{aligned}
$$

Figure 1 shows the cross section of the two cylinders projected on a plane which is perpendiculur to the axis of the cylinders. The limits of integration cover all paths between the cfrcles passing through moderator only. The 1 imiting values of $\theta$ are from $-\frac{\pi}{2}$ to $\frac{\pi}{2}$. The 1 imiting values of

*This expression is erroneously given as $x=d \cos \theta^{\prime}-\cdots-6 \cos$ $\left(\theta+\theta^{\prime}\right)$ in Reference 1 . 
BLANK PAGE T2: 


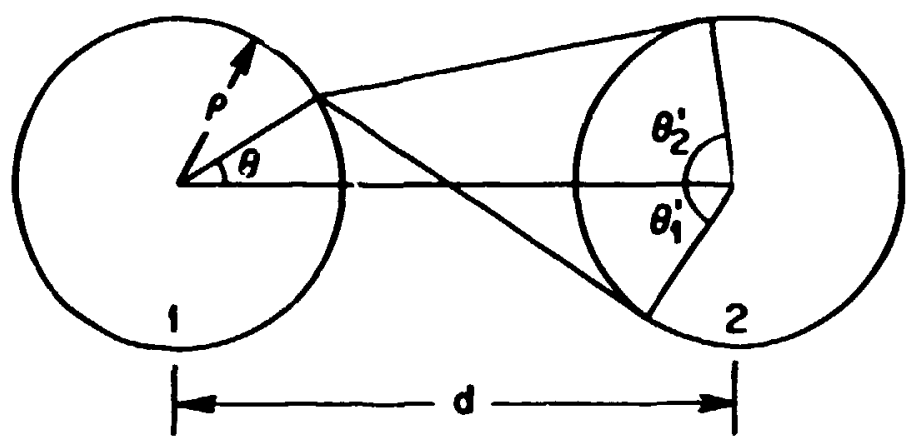

Figure 1. Limits of integration.

$\theta^{\prime}$ for a given $\theta$ wich satisfy the stipulated conditions are from $-\theta_{i}^{\prime}$ to $\theta_{i}$. Expressions for $\theta_{i}$ and $\theta_{2}$ are developed in Appenaix $A$. Equation (1) then becomes:

$$
C=\frac{2 \rho}{\pi^{2}} \int_{0}^{\frac{\pi}{2}} d \theta \int_{\theta_{1}^{\prime}}^{\theta_{2}^{\prime}} d \theta^{\prime} \frac{x x^{\prime}}{\left|\rho^{ \pm}-\rho^{\prime}\right|^{3}} K i_{3}\left(\frac{\left|\rho-\rho^{\prime}\right|}{\lambda}\right) .
$$

Reference 2 gives values of C from Equation (2) in Table 4-25.

In problems of practical interest, we have an array of cylinders instead of the two considered so far. The total Dancoff correction to apply to a given cylinder is the sum of the values of $C$ obtained for all other cylinders in the array. However, for the moderating materials usually encountered, the value of $C$ decreases rapidly with distance. For practical purposes, oniy the nearest and next nearest cylinders give significant contributions to the total correction. For sufficiently close packed arrays, each of these cylinders can be partially obscured from a given unit by adjacent cylinders in the array. When this occurs, the 
limits of integration for Equation (2) must be modified to exclude the obscured portions from the calculations. The top limit of the integral can be reduced from $\frac{\pi}{2}$ to some $\sigma_{\operatorname{Max}}<\frac{\pi}{2}$, and the vaives of $\theta_{1}$ and $\xi_{2}$ can also be modified. The conditions that require these changes and expressions for the corrected ?imits are given in Appendix B for square pitch arrays and in Appendix $C$ for triangular pitch arrays.

When the cylindrical rods in an array are covered with a clad, the path between rods must pass through the clad as well as the moderator. Eince the mean free path of the clad is generally not the same as that of the moderator, that portion of Equation (2) involving the mear. free path must be modified. The effects of clad and gaps are discussed in Appendix D.

A computer program to calculate the Dancoff factor of cylindrical rots in arrays has teen prepared. It uses FORTRAN IV, double precision, and is designed to operate on IBM -360 or IBM-370 series computers. Equation (2), with appropriate modifications discussed here, is calculated by a double numerical integration over the values of $G$ and $G^{\prime}$. A Gaussian quadrature scheme is used to perform the integrations. A higher order of quadrature and closer intervals are used for s,inaller values of 9 , where the contributions to the value of $C$ are larger. As $\theta$ increases, lower orders and somewhat wider interval spacings are used. This procedure allows the calculations to proceed more rapidly. The accuracy of the integrations was tested by varying the order of quadrature and interval spacing for typical problems and observing no significant changs in the results. Values of the $\mathrm{Ki}_{3}(x)$ function were obtained from the rational approximations of Gargantini and Pomentale. 5 Typical rurning times on the IBM-360, 
Hodel 91, are two seconds per array for bare rods and five seconds for rods with clad. 


\section{II . SPHERES}

For two lumps of arbitrary geometry, Dancoff and Ginsburg' give the expression:

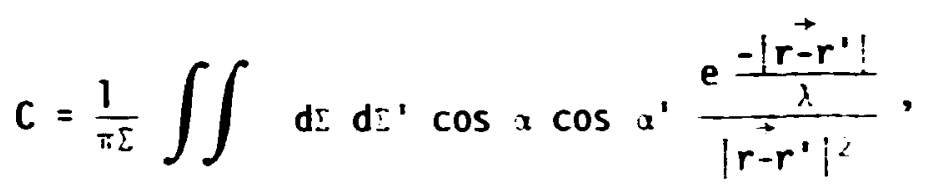

where:

$$
\begin{aligned}
& C=\text { Dancoff factor }, \\
& \ddot{~=~ s u r f a c e ~ a r e a ~ o f ~ f i r s t ~ l u m p, ~} \\
& \check{C}^{\prime}=\text { surface area of second lump, } \\
& x=\text { angle between normal to lump } 1 \text { and the path between lumps, } \\
& x^{\prime} \text { = angle between normal to lump } 2 \text { and the path between lum:s, } \\
& \left|\vec{r}-r^{\prime}\right|=\text { length of a given path between the lumps, } \\
& \lambda=\text { mean free path of the moderator. }
\end{aligned}
$$

Figure 2 shows the cross section of two spheres projected onto a plane passing through the sphere centers. The limits of integration cover all paths between the spheres which pass through moderator only. The element of surface area on sphere $1, d i$, is obtained by considering a circular strip passing through the point $P$. This strip has a radius of $\rho \sin \theta$, a width $\circ d \theta$, and a length $2-n \sin \theta$. Thus:

$$
d \tau=2 \pi n^{2} \sin \theta d \theta \text {. }
$$

Similarly, the element of surface area on sphere 2 is a circular st: ip passing through the point $P^{\prime}$. This strip has a radius of $n \sin (\cdots-r)$ 


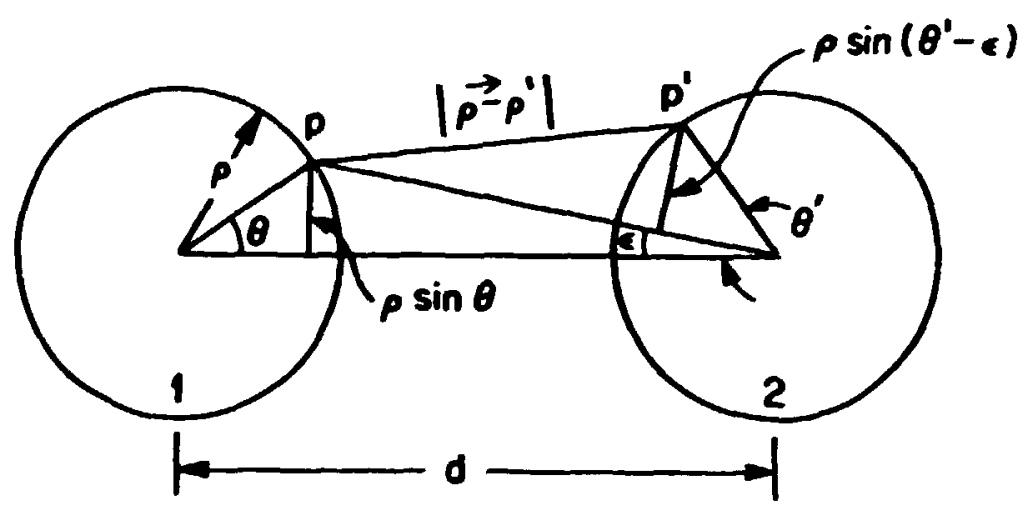

Figure 2. Elements of surface area.

and a width of $\rho d \theta^{\prime}$. For some cases, this strip will be interrupted by the requirement that the path between the spheres must pass through moderator only. If $\phi$ is the angle around the circular path starting from $\phi=0$ at $P^{\prime}$. an angle $\phi_{M} \leq \pi$ is the limiting angle reached. The circular strip length is

$$
2 \int_{0}^{\phi_{M}} \rho \sin \left(\theta^{\prime}-\varepsilon\right) d \phi .
$$

Thus:

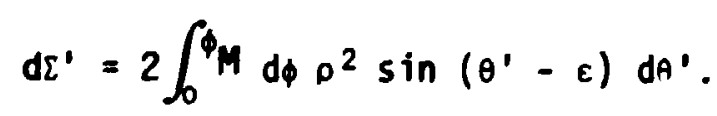

The expression for $\varepsilon$ is given in Appendix $A$, Equation (A2), and the expression for $\phi_{M}$ is given in Appendix E, Equation (E8).

$$
\cos a=\frac{x}{\left|\rho-\rho^{\prime}\right|} \text {, }
$$

where $x$ is given in Appendix E, Equation (ET), and shown in Figure ET, 


$$
\begin{aligned}
& \cos a^{\prime}=\frac{x^{\prime}}{\left|\rho-\partial^{\prime}\right|}, \\
& x^{\prime}=d \cos \theta^{\prime}-\rho-c \cos \left(\theta+\theta^{\prime}\right), \\
& \left|0-\partial^{\prime}\right|=\left|r-r^{\prime}\right|=\sqrt{d^{\prime}-2 d \rho\left(\cos \theta+\cos \theta^{\prime} \mid+20^{2}\left[1+\cos \left(\theta+\theta^{\prime}\right)\right] .\right.}
\end{aligned}
$$

The limits of the $\theta$ integration are from 0 to $\frac{\pi}{2}$, and the limits of the $\theta$ ' integration are from $\varepsilon$ to $\theta_{2}^{\prime}$, where $\theta_{2}^{\prime}$ is the same as for cylinders, Equation (A4).

The integration over values of the angle $\phi$ may be carried out, as shown in Equation (E9). When all of the terms for the spherical geometry are substituted into Equation (3), we get:

$C=\frac{\rho^{2}}{\pi} \int_{0}^{\frac{\pi}{2}} d \theta \int_{\varepsilon}^{\theta^{\prime} \frac{d}{2}} \frac{d \theta^{\prime} \sin \theta \sin \left(\theta^{\prime}-\varepsilon\right)}{\left.|\rho-\rho|^{\prime}\right|^{4}}$

$\left\{\left[d \cos \theta-\rho-\rho \cos (\theta+\varepsilon) \cos \left(\theta^{\prime}-\varepsilon\right)\right] \phi_{M}+\rho \sin (\theta+\varepsilon) \sin \left(\theta^{\prime}-\varepsilon\right)\right.$

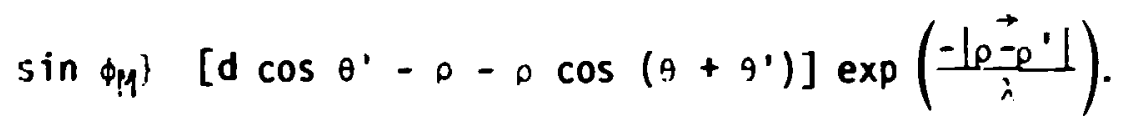

A computer program, which calculates the Dancoff factors of spheres from Equation (5) has been written. It uses FORTRAN IV, double precision, and is designed to ru.1 on IBM-360 or IBM-370 series computers. T'ie double numerical integration over $\theta$ and $\theta^{\prime}$ is done by a Gaussian quadrature scheme. A higher order of quadrature and closer intervals are used for smaller values of $\theta$ where the contributions to the total value of $C$ are larger. As $\theta$ increases, lower orders and somewhat wider interval spacings are used. This procodure allows faster running time on the computer 
8

without loss in accuracy. Typical running times on the IBH-360, Model 91 , are one second per value of $C$.

No attempt has been made to account for the effects of shadowing of spheres in arrays, as was done for cylinders. In addition, the effects of clad and gaps have not been considered. Since spherical arrays are seldom encountered in practice, the extra time and effort did not appear to be justified. For cylindrical arrays, only the nearest and next nearest units were considered. For spherical arrays, the third nearest units are close enough so that they probably should also be included in the total value of $\mathrm{C}$ for an array. 
III. SLAB AKRAYS

Section 4 of ANL $-5800^{2}$ gives the expression for the Dancoff factor of infinite slabs, as follows:

$$
1-C=1-2 \varepsilon_{3}\left(\Sigma_{5} d\right) \text {, }
$$

where:

$$
\begin{aligned}
& C=\text { Dancoff factor, } \\
& \Sigma_{S}=\text { cross section in the moderator, } \\
& d=\text { distance between slabs, } \\
& E_{3}(x)=\text { exponential integral. }
\end{aligned}
$$

This equation may be written in the form:

$$
c=2 t_{3}\left(\frac{d}{\lambda}\right) \text {, }
$$

where $\lambda=$ moderator mean free path. The method for computing the vallies of $E_{j}(x)$ is given in Appendix $F$.

If the slabs in a given array are covered with clad, Equation (7) be':omes:

$$
C=2 E_{3}\left(\frac{d_{m}}{\lambda}+\frac{d_{c}}{\lambda_{c}}\right) \text {, }
$$

where:

$$
\begin{aligned}
& d_{m}=\text { distance through moderator, } \\
& d_{c}=\text { distance through clad, } \\
& \lambda_{c}=\text { mean free path in clad. }
\end{aligned}
$$


10

A computer program to calculate the Dancoff factor of sibs from Equation (8) has been completed. It uses FORTRAN IV, double precision, and is designed to operate on IBM-360 or IBM-370 series computers. Typical running time on the IBM-360, Model 91 , is a fraction of a second per value of $\mathrm{C}$. 


\section{RESULTS}

The values of the Dancoff factors determined by the computer programs have been checked by two methods. The first method was a direct comparison between the computed quantities and existing tables, and the second was a comparison between these computed values and those obtained from Monte Carlo calculations. The values deter..ined for two cylinders were compared with Table 4-25 of Reference 2 over a wide range of parameters, and in all cases the agreement was within the quoted accuracy of the table. The effect of shadowing for cylindrical arrays was checked by comparison with results from a modified version of the MORSE-SCG ${ }^{6}$ Monte Carlo computer program. Test problems in both square pitch and triangular pitch arrays gave saiisfactory agreement. For two spheres, the agreement with Monte Carlo results was also satisfactory. The slab results were checked by comparison with values of the $E_{3}(x)$ function given in Table 5.4, Reference 7 . The agreement here is also good.

Since no previous results for the Dancoff factors of two spheres have been found, a short table of values is given in Table 1. 
TABLE 1. DANCOFF FACTORS FOR SPHERES

\begin{tabular}{|c|c|c|c|c|c|c|c|c|c|c|}
\hline$n \wedge$ & 0. & 0.1 & 0.2 & 0.2 & 0.4 & 0.5 & 0.6 & .7 & .8 & 1.9 \\
\hline $\begin{array}{l}20 \\
30 \\
40 \\
50 \\
60 \\
70 \\
80 \\
90\end{array}$ & $\begin{array}{l}.07559 \\
.06650 \\
.05532 \\
.05340 \\
.04840 \\
.04412 \\
.04042 \\
.03719 \\
.03435 \\
.03183\end{array}$ & $\begin{array}{l}.07296 \\
.04338 \\
-C 5586 \\
.04969 \\
.04453 \\
.64014 \\
.63637 \\
.03310 \\
.03024 \\
.62772\end{array}$ & $\begin{array}{l}.06 \\
.05 \\
.04 \\
. C 4 \\
.03 \\
.03 \\
.02 \\
.02 \\
.02\end{array}$ & $\begin{array}{l}. c 6 n 13 \\
.05769 \\
.04944 \\
.04314 \\
.03779 \\
.03331 \\
.02952 \\
.02428 \\
.02350 \\
.02108\end{array}$ & $\begin{array}{l}.06592 \\
.05511 \\
.04684 \\
.04024 \\
.03495 \\
.03038 \\
.02662 \\
.02345 \\
.02074 \\
.01841\end{array}$ & $\begin{array}{l}.04383 \\
.05248 \\
.04624 \\
.03757 \\
.03216 \\
.02772 \\
.02403 \\
.02093 \\
.01831 \\
.01608\end{array}$ & $\begin{array}{l}.06185 \\
.05039 \\
.04191 \\
.03509 \\
.02970 \\
.02532 \\
.02170 \\
.01870 \\
.01618 \\
.01406\end{array}$ & $\begin{array}{l}.05797 \\
.04924 \\
.03935 \\
.03210 \\
.02745 \\
.02314 \\
.01962 \\
.01572 \\
.01431 \\
.01230\end{array}$ & $\begin{array}{l}.05920 \\
.04621 \\
.03743 \\
.03588 \\
.02539 \\
.02116 \\
.01774 \\
.01496 \\
.01267 \\
.01077\end{array}$ & $\begin{array}{l}.05 \times 51 \\
.04420 \\
.03544 \\
.02872 \\
.02349 \\
.01936 \\
.01606 \\
.01339 \\
.01127 \\
.00944\end{array}$ \\
\hline 3.00 & $\begin{array}{l}.02959 \\
.02759 \\
.02578 \\
.02416 \\
.02268 \\
.02134 \\
.02012 \\
.01500 \\
.01797 \\
.01703\end{array}$ & $\begin{array}{l}.62550 \\
-07352 \\
.02175 \\
.62016 \\
-01874 \\
-C 1746 \\
-C 1627 \\
-61521 \\
. C 1424 \\
.01335\end{array}$ & $\begin{array}{l}. C 2159 \\
.62007 \\
.01836 \\
.01485 \\
.01549 \\
.01427 \\
.01318 \\
.01219 \\
.01125 \\
.01048\end{array}$ & $\begin{array}{l}. C 189 \pi \\
.01714 \\
. C 1552 \\
. C 1409 \\
. C 1282 \\
.01169 \\
.01068 \\
.00977 \\
. c 0896 \\
.00023\end{array}$ & $\begin{array}{l}.01429 \\
.01455 \\
.01312 \\
.01179 \\
.01061 \\
.00558 \\
.00444 \\
.00784 \\
.00712 \\
.00647\end{array}$ & $\begin{array}{l}.01617 \\
.01253 \\
.01111 \\
.00987 \\
.00879 \\
.00785 \\
.00703 \\
.00630 \\
.00566 \\
.00509\end{array}$ & $\begin{array}{l}.01226 \\
.01072 \\
.00961 \\
.00927 \\
.00729 \\
.00544 \\
.00571 \\
.00506 \\
.00450 \\
.00401\end{array}$ & $\begin{array}{l}.01061 \\
.03918 \\
.03797 \\
.00696 \\
.02405 \\
.03579 \\
.03444 \\
.03407 \\
.00358 \\
.00315\end{array}$ & $\begin{array}{l}.00919 \\
.00797 \\
.0067 n \\
.00592 \\
.00503 \\
.00435 \\
.00377 \\
.00329 \\
.00285 \\
.00269\end{array}$ & $\begin{array}{l}.00797 \\
.00675 \\
.00574 \\
.00489 \\
.00418 \\
.00358 \\
.00307 \\
.00244 \\
.00278 \\
.00194\end{array}$ \\
\hline & $\begin{array}{l}.01616 \\
.01535 \\
.01461 \\
.01391 \\
.01321 \\
.01267 \\
.01211 \\
.01154 \\
.011110 \\
.01064\end{array}$ & $\begin{array}{l}.01254 \\
.01179 \\
.01110 \\
.01047 \\
.00588 \\
.00934 \\
.00884 \\
.00837 \\
.60753 \\
.00753\end{array}$ & 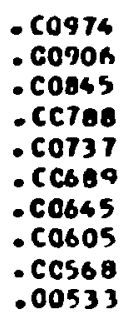 & $\begin{array}{l}. \cos 57 \\
.00697 \\
.00443 \\
.00594 \\
.00550 \\
.00509 \\
.00472 \\
.00430 \\
.00406 \\
.00378\end{array}$ & $\begin{array}{l}.00589 \\
.00537 \\
.00490 \\
.00448 \\
.00410 \\
.00376 \\
.00345 \\
.00317 \\
.00297 \\
.00248\end{array}$ & $\begin{array}{l}.00 \\
.00 \\
.00 \\
.00 \\
.00 \\
.00 \\
.00 \\
.00 \\
.00 \\
.00\end{array}$ & $\begin{array}{l}.00 \\
.00 \\
.00 \\
.00 \\
.00 \\
.00 \\
.00 \\
.00 \\
.00 \\
.00\end{array}$ & $\begin{array}{l}-0 \\
.00 \\
.0 \\
.0 \\
.0 \\
.0 \\
.0 \\
.0 \\
.0\end{array}$ & $\begin{array}{l}.00 \\
.00 \\
.00 \\
.00 \\
.00 \\
.00 \\
.00 \\
.00 \\
.00 \\
.00\end{array}$ & $\begin{array}{l}. \\
.0 \\
.00 \\
.00 \\
.00 \\
.0 \\
.00 \\
.00 \\
.0 \\
.00\end{array}$ \\
\hline & $\begin{array}{l}.01021 \\
.00581 \\
.00942 \\
.005 C 7 \\
.00873 \\
.00941 \\
.00810 \\
.09782 \\
.00755 \\
.00729\end{array}$ & $\begin{array}{l}. \cot 15 \\
. \cos 80 \\
.00447 \\
. \cot 16 \\
-\cos 87 \\
.00560 \\
-\cos 36 \\
-\cos 10 \\
. \cos 87 \\
.00466\end{array}$ & 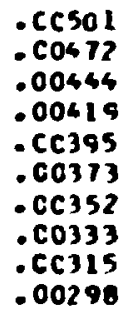 & $\begin{array}{l}. c 0252 \\
.00329 \\
.00305 \\
.00285 \\
. c 0266 \\
.00249 \\
.00232 \\
.02217 \\
.00704 \\
.00191\end{array}$ & $\begin{array}{l}.00247 \\
.00225 \\
.00210 \\
.00194 \\
.00179 \\
.00164 \\
.00154 \\
.00142 \\
.00132 \\
.00122\end{array}$ & $\begin{array}{l}.00 \\
.00 \\
.00 \\
.00 \\
.00 \\
.00 \\
.00 \\
.00 \\
.00 \\
.00\end{array}$ & $\begin{array}{l}.00 \\
.00 \\
.00 \\
.00 \\
.00 \\
.00 \\
.00 \\
.00 \\
.00 \\
.00\end{array}$ & $\begin{array}{l}.00066 \\
.00077 \\
.00769 \\
.00062 \\
.00055 \\
.00050 \\
.00064 \\
.02040 \\
.00036 \\
.00032\end{array}$ & $\begin{array}{l}.00061 \\
.00054 \\
.00047 \\
.00042 \\
.00037 \\
.00 \div 3 ? \\
.00029 \\
.00026 \\
.00023 \\
.00021\end{array}$ & $\begin{array}{l}.00 \\
.00 \\
.00 \\
.00 \\
.00 \\
.00 \\
.00 \\
.00 \\
.00 \\
.00\end{array}$ \\
\hline & & & $\begin{array}{l}.00282 \\
.00267 \\
.00254\end{array}$ & $\begin{array}{l}. \operatorname{col} 79 \\
.00138 \\
.00157\end{array}$ & $\begin{array}{l}.001113 \\
.00105 \\
.00098\end{array}$ & $\begin{array}{l}.00072 \\
.00068 \\
.00061\end{array}$ & $\begin{array}{l}.00046 \\
.00042 \\
.00033\end{array}$ & $\begin{array}{l}.00029 \\
.00026 \\
.00024\end{array}$ & $\begin{array}{l}.00019 \\
.00011 \\
.00015\end{array}$ & $\begin{array}{l}.00 \\
.00 \\
.05\end{array}$ \\
\hline & $\begin{array}{l}.00638 \\
.00618 \\
.00549 \\
.00581\end{array}$ & $\begin{array}{l}-\cos 92 \\
-\cos 75 \\
-\cos 60 \\
\cdot \cos 46\end{array}$ & $\begin{array}{l}.00241 \\
. \operatorname{cc} 228 \\
.00217 \\
.00206\end{array}$ & $\begin{array}{l}. \operatorname{col} 48 \\
.00139 \\
.00131 \\
. \operatorname{col} 23\end{array}$ & $\begin{array}{l}.00091 \\
.00005 \\
.00079 \\
.00073\end{array}$ & $\begin{array}{l}.00056 \\
.00052 \\
.00048 \\
.00044\end{array}$ & $\begin{array}{r}.00035 \\
.00031 \\
.00029 \\
.00026\end{array}$ & $\begin{array}{l}.00021 \\
.00019 \\
.00117 \\
.00016\end{array}$ & $\begin{array}{l}.00013 \\
.00012 \\
.00011 \\
.00009\end{array}$ & $\begin{array}{l}.0000 A \\
.00007 \\
.00006 \\
.00006\end{array}$ \\
\hline & $\begin{array}{r}.00543 \\
.00547 \\
.00531\end{array}$ & $\begin{array}{l}.00332 \\
.00319 \\
.00307\end{array}$ & $\begin{array}{l}.00196 \\
.00186 \\
.00171\end{array}$ & $\begin{array}{l}.00116 \\
.00109 \\
.00103\end{array}$ & $\begin{array}{l}.00068 \\
.00064 \\
.00059\end{array}$ & $\begin{array}{r}.00040 \\
.00037 \\
.00034\end{array}$ & $\begin{array}{l}.00024 \\
.00022 \\
.00020\end{array}$ & $\begin{array}{l}.00016 \\
.010013 \\
.00012\end{array}$ & $\begin{array}{l}.000 \cap 8 \\
.00006 \\
.00001\end{array}$ & $\begin{array}{l}.00005 \\
.00004 \\
.00004\end{array}$ \\
\hline
\end{tabular}

$D=$ Pitch.

Rho = Sphere radius.

Lambda = Moderator mean free path. 
TABLE :. (cont.)

\begin{tabular}{|c|c|c|c|c|c|c|c|c|c|c|}
\hline 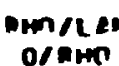 & & & & 0.3 & 4 & .5 & 6 & 7 & 8 & 9 \\
\hline $\begin{array}{l}7.00 \\
7.10 \\
7.20 \\
7.30 \\
7.40 \\
7.50 \\
7.60 \\
7.70 \\
7.80 \\
7.90\end{array}$ & $\begin{array}{l}00516 \\
005 c 1 \\
00487 \\
00476 \\
.00461 \\
.00442 \\
00437 \\
00425 \\
.00414\end{array}$ & $\begin{array}{l}. c 0295 \\
.00286 \\
.00213 \\
.00263 \\
.00253 \\
.00244 \\
.00235 \\
.00227 \\
.00219 \\
.00211\end{array}$ & $\begin{array}{l}. c 2169 \\
-c 0161 \\
.00153 \\
.00146 \\
.00139 \\
.00133 \\
.06127 \\
.06121 \\
.00115 \\
.00110\end{array}$ & $\begin{array}{l}-60097 \\
-00091 \\
-(0086 \\
.00081 \\
-0007 ? \\
-00072 \\
-00068 \\
-00064 \\
-00061 \\
-\cos 1\end{array}$ & $\begin{array}{l}.00055 \\
.00052 \\
.00048 \\
.00045 \\
.00042 \\
.00039 \\
.00037 \\
.00034 \\
.00032 \\
.00030\end{array}$ & $\begin{array}{l}.00 \\
.00 \\
.00 \\
.00 \\
.00 \\
.00 \\
.00 \\
.00 \\
.00 \\
.00\end{array}$ & $\begin{array}{l}.00018 \\
.00011 \\
.00015 \\
.00011 \\
.00013 \\
.00012 \\
.00012 \\
.00010 \\
.00009 \\
.00008\end{array}$ & $\begin{array}{l}.0 \\
.0 \\
.0 \\
.0 \\
.0 \\
.0 \\
.0 \\
.0 \\
.0 \\
.0\end{array}$ & $\begin{array}{l}26 \\
05 \\
05 \\
06 \\
06 \\
06 \\
03 \\
33 \\
03 \\
22\end{array}$ & $\begin{array}{l}.0 \\
.0 \\
.0 \\
.0 \\
.0 \\
.0 \\
.0 \\
.0\end{array}$ \\
\hline $\begin{array}{l}. .00 \\
6.10 \\
. .20 \\
0.30\end{array}$ & $\begin{array}{l}.00 \\
.00 \\
.00 \\
.00 \\
.00 \\
.00 \\
.00 \\
.00 \\
.02 \\
.00\end{array}$ & $\begin{array}{l}. c 0 \\
. c 0 \\
. c 0 \\
.00 \\
. c 0 \\
. c 0 \\
. c 0 \\
. c 0 \\
. c 0 \\
.6 C\end{array}$ & $\begin{array}{l}.00 \\
.00 \\
.0 C \\
.00 \\
.0 C \\
.00 \\
.0 C\end{array}$ & $\begin{array}{l}. \mathrm{CC} \\
.00 \\
.00 \\
.00 \\
. \mathrm{CO} \\
. \mathrm{CO} \\
. \mathrm{Cc}\end{array}$ & $\begin{array}{l}.00 \\
.02 \\
.00 \\
.00 \\
.00 \\
.00 \\
.00 \\
.00\end{array}$ & $\begin{array}{l}.00 \\
.00 \\
.00 \\
.00 \\
.00 \\
.06 \\
.00 \\
.00 \\
.00 \\
.00\end{array}$ & $\begin{array}{l}.0 \\
.0 \\
.0 \\
.0 \\
.0 \\
.0 \\
.0 \\
.0 \\
.0 \\
.0\end{array}$ & $\begin{array}{l}.0 \\
.0 \\
.0 \\
.0 \\
.0 \\
.0 \\
.0 \\
.1 \\
.0 \\
.0\end{array}$ & $\begin{array}{l}2 \\
32 \\
22 \\
21 \\
01 \\
21 \\
11 \\
11 \\
01\end{array}$ & $\begin{array}{l}.0 \\
.0 \\
.0 \\
.0 \\
.0 \\
.0 \\
.0\end{array}$ \\
\hline 9.00 & $\begin{array}{l}.00311 \\
.00334 \\
.00257 \\
.00251 \\
.00285 \\
.00279\end{array}$ & $\begin{array}{l}. c C 145 \\
.00141 \\
.00136 \\
.00132 \\
. \operatorname{col} 28 \\
. \operatorname{col} 24 \\
. \operatorname{col} 20 \\
. \operatorname{col} 116 \\
.00113 \\
.00109\end{array}$ & $\begin{array}{l}68 \\
65 \\
52 \\
60 \\
57 \\
55 \\
53 \\
51 \\
49 \\
47\end{array}$ & $\begin{array}{l}.6 \alpha \\
. c \alpha \\
.00 \\
.00 \\
.00 \\
.00 \\
.00 \\
. c 0 \\
.00\end{array}$ & $\begin{array}{l}.00 \\
.00 \\
.00 \\
.00 \\
.00 \\
.00 \\
.00 \\
.00 \\
.00 \\
.00\end{array}$ & $\begin{array}{l}.0 \\
.0 \\
.0 \\
.0 \\
.0 \\
.0 \\
.0 \\
.0\end{array}$ & $\begin{array}{l}.00003 \\
.00003 \\
.00003 \\
.00003 \\
.00002 \\
.00002 \\
.00002 \\
.00002 \\
.00002 \\
.00002\end{array}$ & $\begin{array}{l}.0 \\
.0 \\
.0 \\
.0 \\
.0 \\
.0 \\
.0 \\
.0 \\
.0 \\
.0\end{array}$ & $\begin{array}{l}01 \\
01 \\
01 \\
01 \\
0.3 \\
00 \\
00 \\
00 \\
00 \\
00\end{array}$ & \\
\hline $\begin{array}{l}10 \\
10 \\
10\end{array}$ & $\begin{array}{l}.00228 \\
.00224 \\
.00219 \\
.00215 \\
.0021 !\end{array}$ & $\begin{array}{l}c 0166 \\
c 0103 \\
c 0100 \\
c 0057 \\
c 0094 \\
c 0092 \\
c 0089 \\
c 0086 \\
c 0084 \\
0 n 082\end{array}$ & $\begin{array}{l}. c c 045 \\
.00043 \\
.00041 \\
.00040 \\
.00038 \\
.00037 \\
.00035 \\
.00034 \\
.00033 \\
. r 0032\end{array}$ & $\begin{array}{l}.00015 \\
.00014 \\
.00013 \\
.00013 \\
.00012\end{array}$ & $\begin{array}{l}.00009 \\
.00009 \\
.00007 \\
.00007 \\
.00004 \\
.00006 \\
.00006 \\
.00005 \\
.00055 \\
.00005\end{array}$ & $\begin{array}{l}.00003 \\
.00003 \\
.00003 \\
.00003 \\
.00003 \\
.00002 \\
.00002 \\
.00002 \\
.09012 \\
.00702\end{array}$ & $\begin{array}{l}.00001 \\
.00001 \\
.00001 \\
.00001 \\
.00001 \\
.00001 \\
.0001 \\
.00001 \\
.00001 \\
.00001\end{array}$ & $\begin{array}{l}.00001 \\
.00001 \\
.00001 \\
.00000 \\
.00000 \\
.00000 \\
.00000 \\
.00000 \\
.00000 \\
.00000\end{array}$ & $\begin{array}{l}.00000 \\
.00000 \\
.00000 \\
.00000 \\
.00000 \\
.00000 \\
.00000 \\
.00000 \\
.00000 \\
.00000\end{array}$ & $\begin{array}{l}.0,0000 \\
.00000 \\
.00000 \\
.00000 \\
.00000 \\
.00000 \\
.00000 \\
.00000 \\
.00000 \\
.00000\end{array}$ \\
\hline
\end{tabular}


TABLE ?. (cont.)

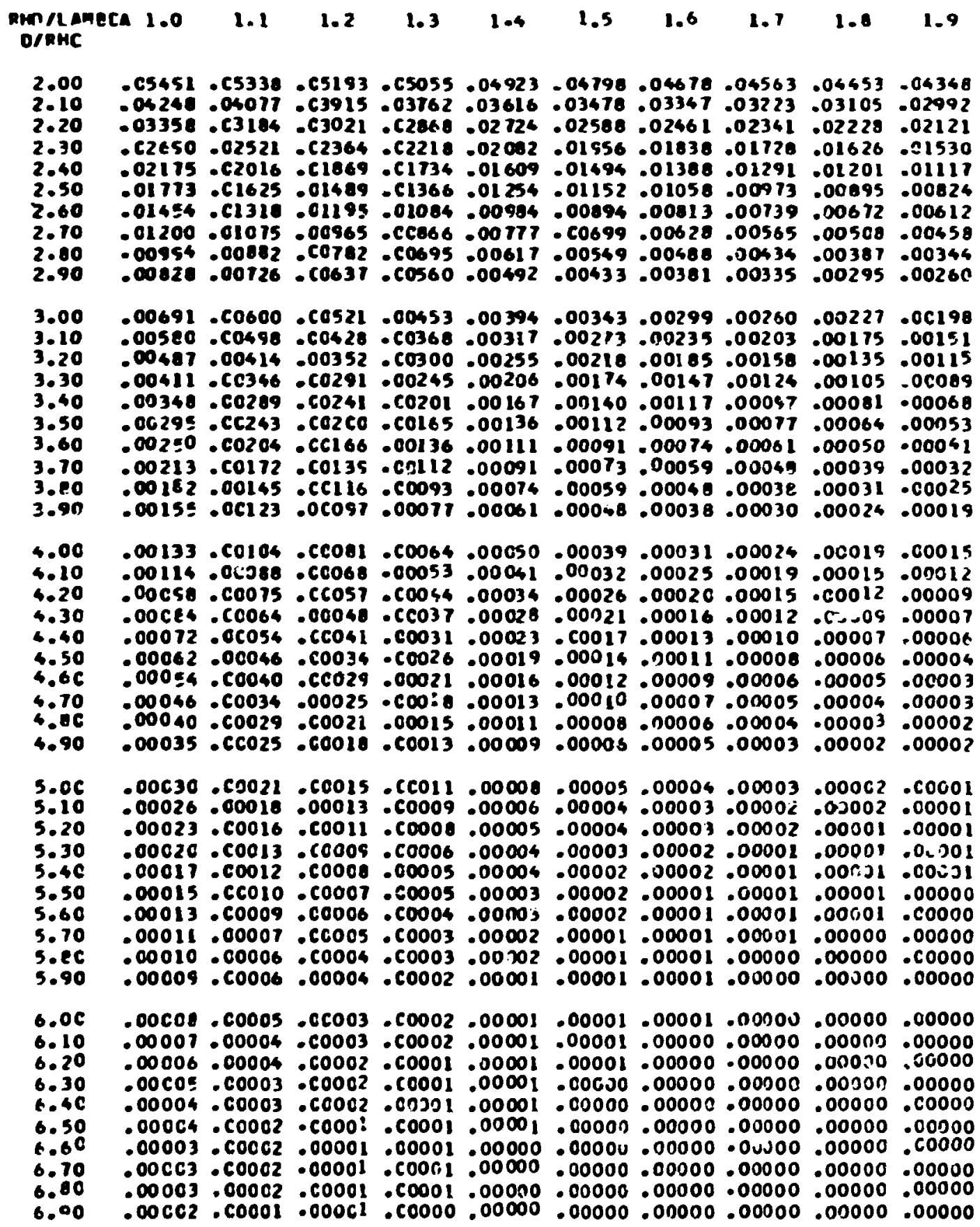


TABLE 1. (cont.)

\begin{tabular}{|c|c|c|c|c|c|c|c|c|c|c|}
\hline $\begin{array}{l}\text { Im / LAMEC } \\
\text { D/RHO }\end{array}$ & 2.0 & 2.1 & 2.2 & 2.3 & 2.4 & 2.5 & 2.6 & 2.7 & 2.8 & 2.9 \\
\hline $\begin{array}{l}2.00 \\
2.10 \\
2.20 \\
2.30 \\
2.40 \\
2.50 \\
2.60 \\
2.70 \\
2.80 \\
2.90\end{array}$ & $\begin{array}{l}.04248 \\
.02885 \\
.02621 \\
.01441 \\
.01040 \\
.00758 \\
.00557 \\
.00413 \\
.00307 \\
.00230\end{array}$ & $\begin{array}{l}C 4152 \\
. C 2183 \\
-61526 \\
. C 1357 \\
-C 0969 \\
-C C 659 \\
-C C 5 C 8 \\
. C 0372 \\
-\therefore C 274 \\
-C 0203\end{array}$ & $\begin{array}{l}. C 4059 \\
.02686 \\
.01936 \\
.61279 \\
.00903 \\
.00844 \\
.00463 \\
.00335 \\
.00244 \\
.00179\end{array}$ & $\begin{array}{l}.03971 \\
.02593 \\
.01752 \\
.01206 \\
.00842 \\
.00594 \\
.06422 \\
.00303 \\
.00218 \\
.00158\end{array}$ & $\begin{array}{l}.03955 \\
.02504 \\
.01672 \\
.03133 \\
.00785 \\
.00548 \\
.00385 \\
.00273 \\
.00195 \\
.00140\end{array}$ & $\begin{array}{l}.03804 \\
.02420 \\
.01596 \\
.01074 \\
.00733 \\
.00505 \\
.00352 \\
.00247 \\
.00174 \\
.00123\end{array}$ & $\begin{array}{l}.03 \\
.02 \\
.01 \\
.01 \\
.00 \\
.00 \\
.00 \\
.00 \\
.00 \\
.00\end{array}$ & $\begin{array}{l}.0 \\
.0 \\
.0 \\
.0 \\
.0 \\
.0 \\
.00 \\
.0 \\
.0 \\
.0\end{array}$ & $\begin{array}{l}.0 \\
.0 \\
.0 \\
.0 \\
.0 \\
.0 \\
.0 \\
.0 \\
.0 \\
.0\end{array}$ & $\begin{array}{l}.03507 \\
.02117 \\
.01330 \\
.00855 \\
.00558 \\
.00368 \\
.06245 \\
.00165 \\
.00111 \\
.00076\end{array}$ \\
\hline $\begin{array}{l}3.00 \\
3.10 \\
3.20 \\
3.30\end{array}$ & $\begin{array}{l}.00173 \\
.00136 \\
.00055 \\
.00075\end{array}$ & $\begin{array}{l}C 0151 \\
.00112 \\
.00084 \\
. C 5083\end{array}$ & $\begin{array}{l}. c 0132 \\
-0 \cos 7 \\
-\operatorname{co0} 72 \\
. c 0054\end{array}$ & $\begin{array}{l}. c 0115 \\
.00084 \\
.00062 \\
. c 0045\end{array}$ & $\begin{array}{l}.00101 \\
.00073 \\
.00053 \\
.00038\end{array}$ & $\begin{array}{l}.00088 \\
.00063 \\
.00045 \\
.00033\end{array}$ & $\begin{array}{l}.00077 \\
.00055 \\
.00039 \\
.00028\end{array}$ & $\begin{array}{l}.00067 \\
.00047 \\
.00033 \\
.00023\end{array}$ & $\begin{array}{l}.00059 \\
.00041 \\
.00029 \\
.00020\end{array}$ & $\begin{array}{l}.00052 \\
.00036 \\
.00024 \\
.00017\end{array}$ \\
\hline $\begin{array}{l}3.40 \\
3.50\end{array}$ & $\begin{array}{l}.00057 \\
.07044\end{array}$ & $\begin{array}{l}-\operatorname{cco} 48 \\
. \operatorname{cro} 36\end{array}$ & $\begin{array}{l}\cdot c \cos 0 \\
-\cos 30\end{array}$ & $\begin{array}{l}.00034 \\
.00025\end{array}$ & $\begin{array}{l}.0002 \mathrm{~B} \\
.0002 \mathrm{I}\end{array}$ & $\begin{array}{l}.00024 \\
.00017\end{array}$ & & & & $\begin{array}{l}.00012 \\
.00008\end{array}$ \\
\hline $\begin{array}{l}3.60 \\
3.70 \\
3.80 \\
3.90\end{array}$ & $\begin{array}{l}.00033 \\
.00026 \\
.00020 \\
.00015\end{array}$ & $\begin{array}{l}. c 0027 \\
. c 0021 \\
.00016 \\
.00012\end{array}$ & $\begin{array}{l}c 0022 \\
+c \operatorname{col} 11 \\
.00013 \\
. c 0010\end{array}$ & $\begin{array}{l}.00018 \\
.00014 \\
.00010 \\
. c 0008\end{array}$ & $\begin{array}{l}.00015 \\
.00011 \\
.0000 B \\
.00006\end{array}$ & $\begin{array}{l}.00012 \\
.00009 \\
.00007 \\
.00005\end{array}$ & $\begin{array}{l}.00010 \\
.00007 \\
.00005 \\
.00004\end{array}$ & $\begin{array}{l}.00508 \\
.00006 \\
.00004 \\
.00003\end{array}$ & $\begin{array}{l}.00007 \\
.00005 \\
.00003 \\
.00002\end{array}$ & $\begin{array}{l}.01006 \\
.00004 \\
.00003 \\
.00002\end{array}$ \\
\hline $\begin{array}{l}4.00 \\
4.10 \\
4.20 \\
4.30 \\
4.40 \\
4.30 \\
4.60 \\
4.70 \\
4.80 \\
4.90\end{array}$ & $\begin{array}{l}.00012 \\
.00009 \\
.00 C C 7 \\
.00 C C 5 \\
.000 C 4 \\
.00003 \\
.00 C C 3 \\
.00002 \\
.00 C 02 \\
.00001\end{array}$ & 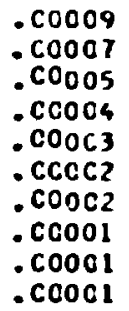 & $\begin{array}{l}.00007 \\
.00006 \\
.00004 \\
.00063 \\
.0000 ? \\
.0000 ? \\
.00001 \\
.00001 \\
.000 C 1 \\
.00001\end{array}$ & $\begin{array}{l}. c 000, \\
.00004 \\
.00003 \\
.60002 \\
.00002 \\
.00001 \\
.00001 \\
.00001 \\
.00001 \\
.00000\end{array}$ & $\begin{array}{l}.00005 \\
.00001 \\
.00002 \\
.00002 \\
.00001 \\
.00001 \\
.00001 \\
.00001 \\
.00000 \\
.00000\end{array}$ & $\begin{array}{l}.00004 \\
.00003 \\
.00012 \\
.00001 \\
.00001 \\
.00001 \\
.00511 \\
.00000 \\
.00000 \\
.00000\end{array}$ & $\begin{array}{l}.00003 \\
.00002 \\
.00001 \\
.00001 \\
.00001 \\
.00001 \\
.00000 \\
.00000 \\
.00000 \\
.00000\end{array}$ & $\begin{array}{l}.00002 \\
.00302 \\
.00001 \\
.00001 \\
.00001 \\
.00 J 00 \\
.00000 \\
.00000 \\
.00000 \\
.00000\end{array}$ & $\begin{array}{l}.00001 \\
.00001 \\
.00001 \\
.00001 \\
.00000 \\
.00000 \\
.00000 \\
.0001,0 \\
.00000 \\
.00000\end{array}$ & $\begin{array}{l}\text {-nnu01 } \\
-00001 \\
.00001 \\
.00000 \\
.00000 \\
.00000 \\
.00000 \\
.00000 \\
.00000 \\
.00000\end{array}$ \\
\hline
\end{tabular}


17

ACKNOWLEDGMENTS

The author wishes to acknowledge the generous assistance of L. M. Petrie in preparing and checking these computer programs. He made the necessary modifications to the MORSE-SGC ${ }^{6}$ Monte Carlo program and performed the calculations used to verify the correctness of the Dancoff factors generated. He also supplied the derivation of the expression for $\cos \alpha$ for spheres given in Appendix E. R. M. Westfall offered many helpful suggestions and supplied the routines used to calculate the $\mathrm{Ki}_{3}$ function.' 


\section{REFERENCES}

1. S. M. Dancoff and M. Ginsburg, "Surface Resonance Abscrption in a Close-Packed Lattice," CP-2157 (September, 1944).

"Argonne National Laboratory, "Reactor Physics Constarts," ANL-5800 (July, 1963).

3. A. Sauer, Nucl. Sci. Eng., 16,329 (1963).

4. H. G. Bickley and J. Naylor, Phil. Mag., 20, Series F, 343 (1935).

5. I. Gargant ini and T. Ponentale, Communicaticns of the ACM, 7, No. 12, 727 (1964).

6. S. K. Fraley, "Users" Guide to MORSE-SCC," ORNL/CSD-7 (March, 1976).

7. M. Abrämorvitz and I. A. Stegun, "Handbook of Mathematical Functions," National Bureau of Standards, AME $5 j$ (June, 1964). 


\section{APPENDIXES}


APPENDIX A

\section{CYLINOER JANCOFF LIMITS OF INTEGRATION}

Consider two cylinders of radius $c$ and having a center-to-center distance (pitch) d. The angle between a given radius and the line connecting the centers is $e$ in cylinder 1 and $\theta^{\prime}$ in cylinder 2 . For a given angle $\theta$ in cylinder 1 , the limits of integration in cylinder 2 are $y_{1}^{1}$ and $e_{2}$. These limits are the largest angle below and above the line of centers ior which the path of neutrons between the cylinder passes only through moderator. This condition is met when the neutron path is tangent to either cylinder 1 or $c y l$ inder 2 . For small values of $\because$, this path is tangent to cylinder 2: and for large values of $\theta$, it is tangent to cyli.nJer 1 . As a limiting value, $\theta_{\mathrm{L}}$, the path is tangent to both cylinders. The expression for $E_{L}$ may be obtained from figure $A I$.

ORNL- DWG 77-20368

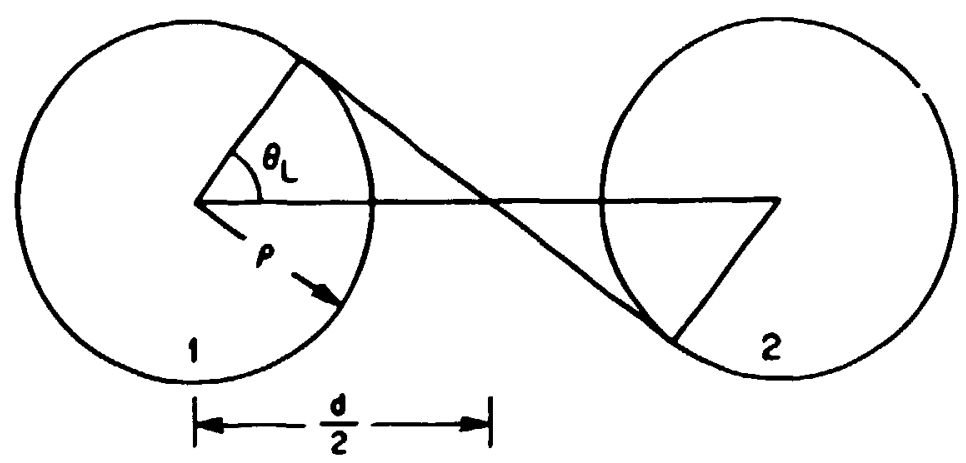

Figure Al. Limiting angle "L.

Due to symmetry, the distance from the intersection of the neutron path with the line of centers to the center of either cylinder is $\frac{d}{2}$. 
Thus:

$$
\cos \theta_{L}=\frac{2 \rho}{d} .
$$

The case for $\theta$ less than $\theta_{L}$ is considered in Fiqure A2.

\section{ORNL- DWG 77-20369}

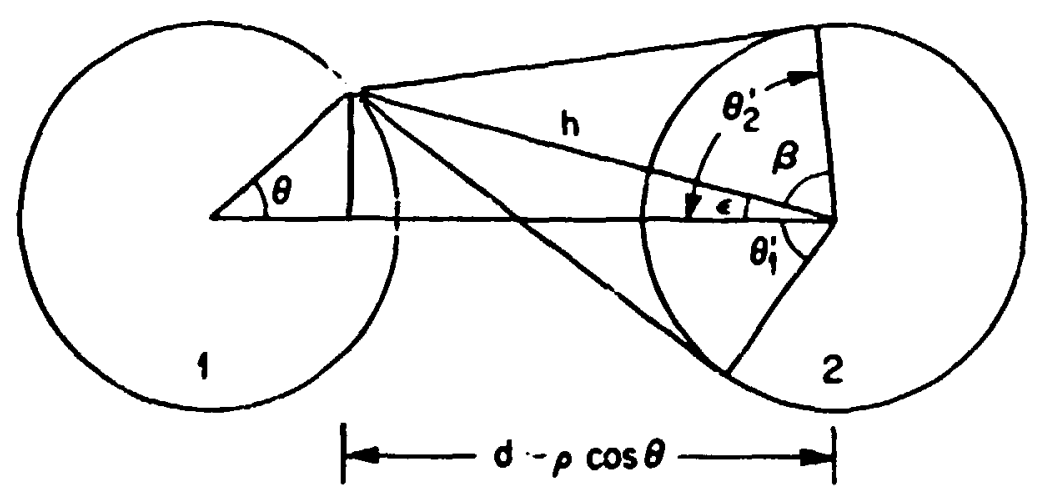

Figure A2. $\theta$ less than $\theta_{L}$.

The distance $h$ from a point at angle $\theta$ on cylinder 1 to the center of cylinder 2 is:

$$
\begin{aligned}
& h=\sqrt{(d-\rho \cos \theta)^{2}+(\rho \sin \theta)^{2},} \\
& h=\sqrt{d^{2}-2 d \rho \cos \theta+\rho^{2}} . \\
& \sin \varepsilon=\frac{\rho \sin \theta}{h}, \cos \beta=\frac{\rho}{h} . \\
& \theta_{1}^{\prime}=\beta-\varepsilon, \theta_{2}^{\prime}=\beta+\varepsilon .
\end{aligned}
$$


Thus:

$$
\begin{aligned}
& \theta_{1}^{\prime}=\cos ^{-1}\left(\frac{\rho}{h}\right)-\sin ^{-1}\left(\frac{\rho \sin \theta}{h}\right), \\
& \theta_{2}^{\prime}=\cos ^{-1}\left(\frac{\partial}{h}\right)+\sin ^{-1}\left(\frac{\rho \sin \theta}{h}\right) .
\end{aligned}
$$

Next, consider the case for $\theta$ greater than $\theta_{L}$, shown in Figure A3.

ORNL- DWG 77-20373

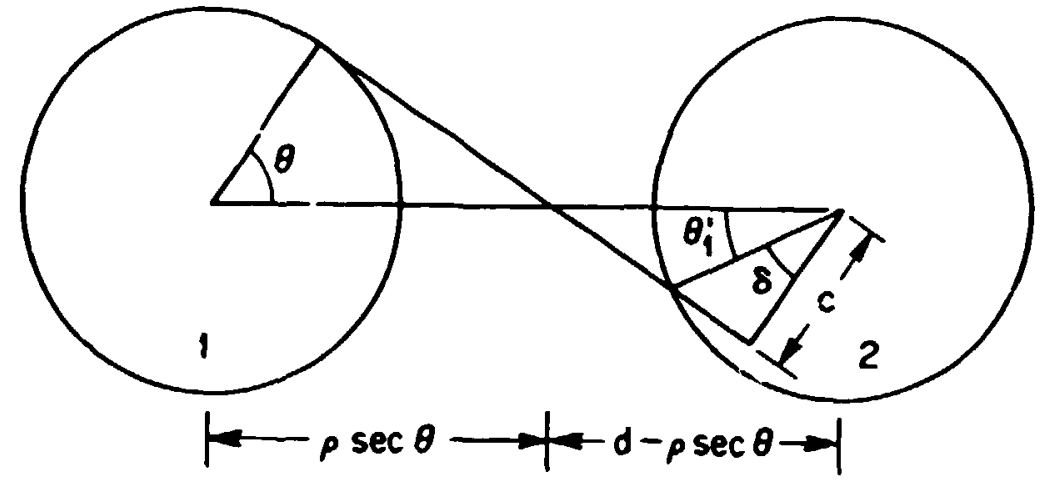

Figure A3. $\theta$ greater than $\theta_{L}$.

$$
\begin{aligned}
& \theta=\theta_{j}+\delta . \\
& c=(d-c \sec \theta) \cos \theta, \\
& c=d \cos \theta-\rho . \\
& \cos \delta=\frac{c}{\rho}=\frac{d \cos \theta}{\rho}-1 . \\
& \theta_{i}^{\prime}=\theta-\delta=\theta-\cos ^{-1}(d \cos \theta-1) .
\end{aligned}
$$

The expression for $\theta_{2}^{\prime}$ is the same as for $\theta$ less than $\theta_{L}$. If $\theta=\theta_{L}$, either expression for $\theta \mathrm{i}$ is correct. 


\section{APPENDIX B \\ CHANGES IN THE LIMITS OF INTEGRATION FOR \\ SQUARE PITCH ARRAYS OF CYLINDERS DUE TO SI'ADOHIHG EFFECTS}

Consider a square array of cylinders of radius $\rho$ and having a pitch $d$, shown in Figure Bi.

$$
\text { ORNL-DWG 77-20371 }
$$

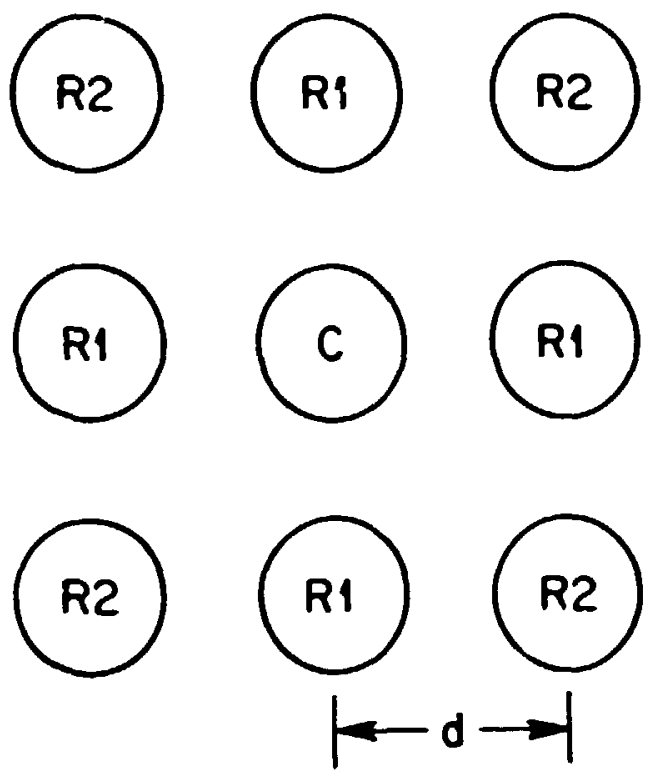

Figure BI. Square array.

For purposes of computing the Dancoff factor, only the four nearest cylinders, designated $R 1$, and the four next nearest cyl inders, designated R2, are considered. The $l$ imits of integration, $\theta_{1}^{\prime}$ and $\theta_{2}^{\prime}$, developed in Appendix $A$ are correct for cylinders R1. However, for sufficiently close packed arrays, cylinders R2 will be partially obscured by cylinders RI, and the expressions for $\theta_{i}$ and $\theta_{2}^{\prime}$ must be modified. In addition, the top limit for the $\theta$ integration (above which none of cylinders $R 2$ can be "seen" only through moderator) must be reduced from $\frac{\pi}{2}$ to some $\theta_{\operatorname{Max}}<\frac{\pi}{2}$. 


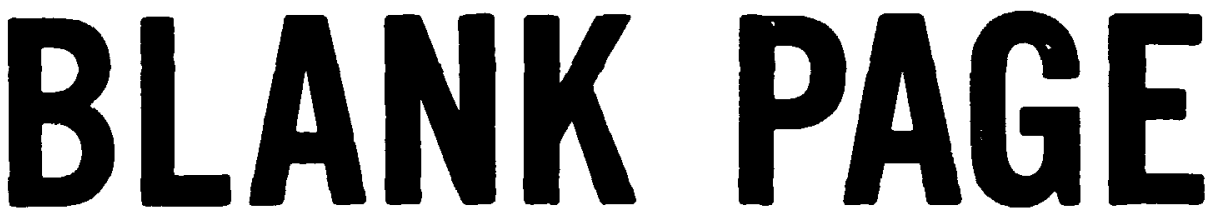


The limiting spacing for shadowing of $\theta_{2}^{\prime}$ is show in Figure B2.

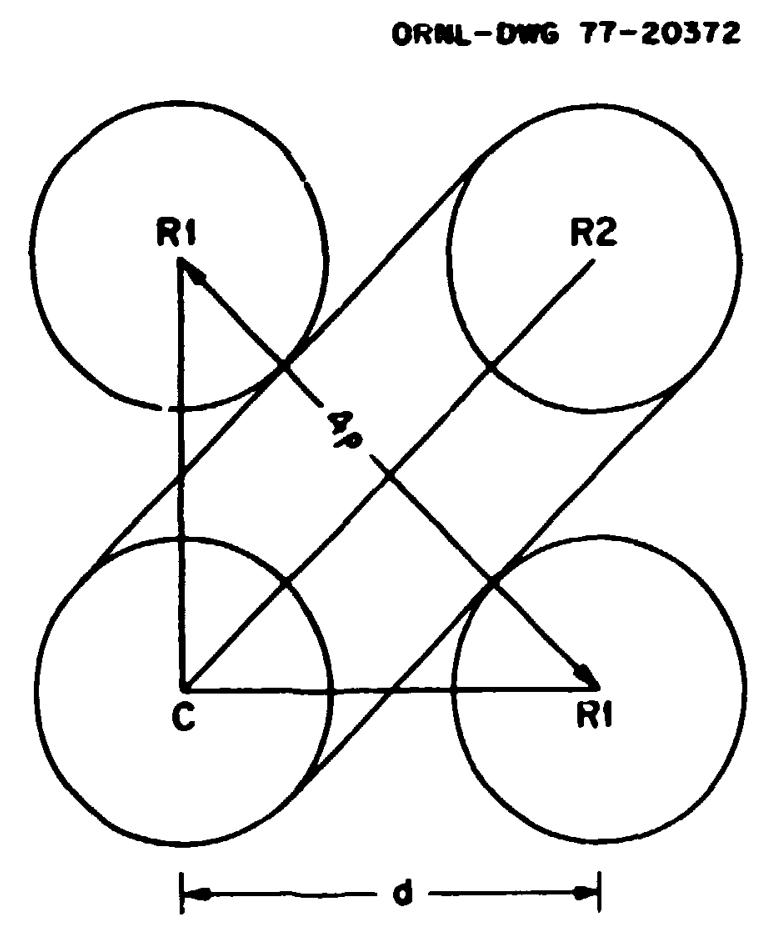

Figure B2. Limiting spacing for shadowing of $\theta_{2}^{\prime}$.

From Figure $B 2$, it can be seen that:

$$
\begin{aligned}
& \sqrt{2} d=4 \rho, \\
& \frac{d}{0}=\frac{4}{\sqrt{2}} .
\end{aligned}
$$

For $\frac{d}{0}<\frac{4}{\sqrt{2}}$, and sufficiently large values of $\theta, \theta_{2}^{\prime}$ must be modified from the expression given in Appendix A. 
Next, consider the limiting spacing for $e_{1}$ shadowing, shown in Figure $B 3$.

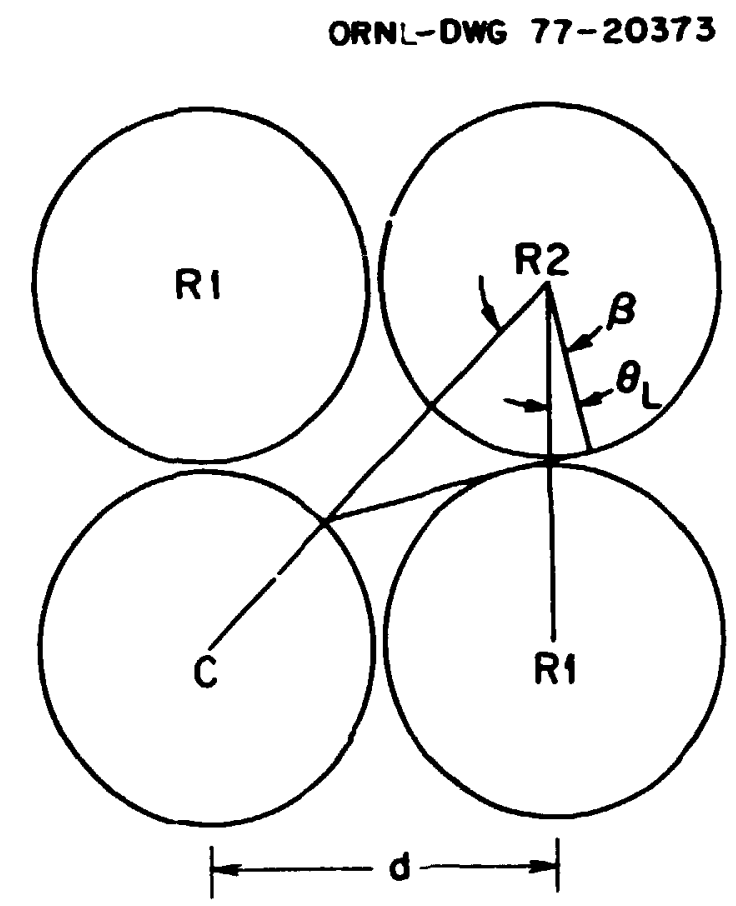

Figure $\mathrm{B}$. Limiting spacing for $\theta i$ shadowing.

From Equation (A1), $\theta_{L}=\cos ^{-1}\left(\frac{2 \rho}{d}\right)$.

Thus:

$$
B=\frac{\pi}{4}+\theta_{L}=\frac{\pi}{4}+\cos ^{-1}\left(\frac{2 D}{d}\right) .
$$


The value of $B$ can also be obtained from Enuations (A2) and (A3) for an angle $\theta=0$ and the distance of separation $=\sqrt{2} d\left(\theta<\theta_{L}\right)$ :

$$
\begin{aligned}
& h=\sqrt{2 d^{2}-2 \sqrt{2} d \rho+\rho^{2}}, \\
& h=\sqrt{2} d-\rho, \\
& h=\cos ^{-1}\left(\frac{\rho}{\sqrt{2} d-\rho}\right) .
\end{aligned}
$$

For the limiting separations considered here, these two expressions for $B$ must be equal.

$$
\frac{\pi}{4}+\cos ^{-1}\left(\frac{2 \rho}{d}\right)=\cos ^{-1}\left(\frac{\rho}{\sqrt{2} d-\rho}\right) .
$$

Noting that:

$$
\sin ^{-1} \sqrt{1-\frac{4 p^{2}}{d^{2}}}=\cos ^{-1} \cdot\left(\frac{20}{d}\right)
$$

and using the expression for the cosine of the sum of two angles leads to:

$$
\begin{aligned}
& \frac{1}{\sqrt{2}}\left(\frac{2 \rho}{d}\right)-\frac{1}{\sqrt{2}} \sqrt{1-\frac{4 \rho^{2}}{d^{2}}}=\frac{\rho}{\sqrt{2} d-\rho}, \\
& \frac{1}{\sqrt{2}}\left(\frac{2}{d / \rho}\right)-\frac{1}{\sqrt{2}} \sqrt{1-\frac{4}{(d / \rho)^{2}}}=\frac{1}{\sqrt{2} d / \rho-1} .
\end{aligned}
$$

This expression was solved for $\frac{d}{\rho}$ by an iterative procedure. Frun this:

$$
\frac{d}{\rho} \simeq 2.0558 \text {. }
$$


For values of $\frac{d}{c}$ less than this, and for sufficiently small values of $\theta$, $\theta_{i}$ will be shadowed and must be modified.

The limiting values of 9 for $g_{2}^{\prime}$ shadowing $\left(\frac{d}{\rho}<\frac{4}{\sqrt{2}}\right)$ are shown in Figure B4.

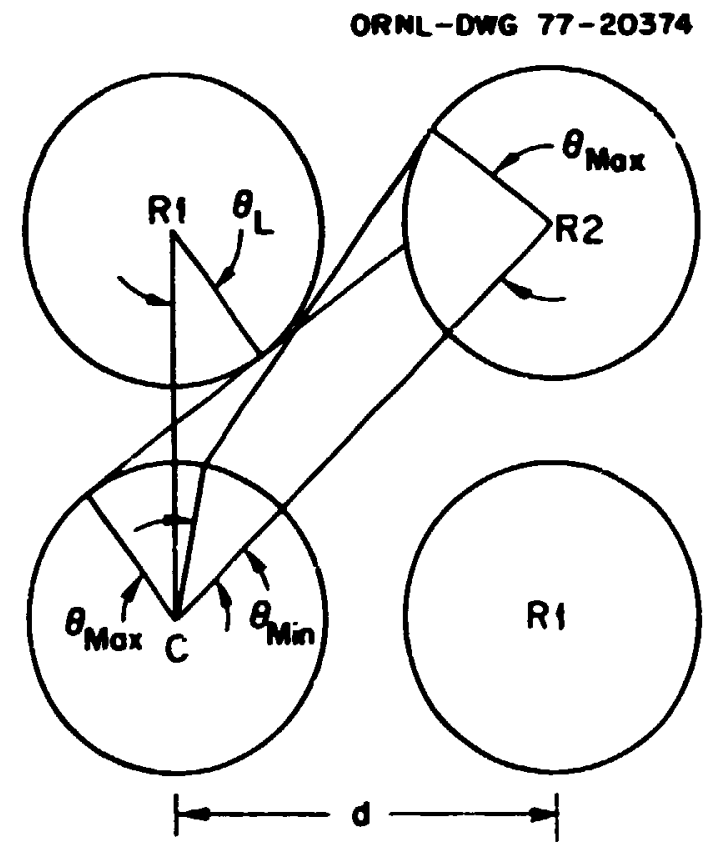

Figure $B 4$. Limiting values of $\theta$ for $e_{2}^{\prime}$ shadowing.

From Equation $(A I), \hat{O}_{L}=\cos ^{-1}\left(\frac{20}{d}\right)$.

$$
\theta_{\operatorname{Max}}=\frac{\pi}{4}+\theta_{L}=\frac{\pi}{4}+\cos ^{-1}\left(\frac{\hat{z}_{p}}{d}\right) .
$$

For values of $\theta \geq \theta_{\text {Max }}$, cylinder $R 2$ is totality obscured from $C$. Thus, the upper limit of the $\theta$ integration is reduced from $\frac{\pi}{2}$ to $\theta_{\text {Max }}$. The expression 
for $\theta_{\text {Kin }}$ may be obtained from Equation (A5) for an angle FMax and a distance of $\sqrt{2} d$ :

$$
\theta_{\operatorname{Min}}=-\left[\theta_{\operatorname{Max}}-\cos ^{-1}\left(\frac{\sqrt{2} d}{\rho} \cos \theta_{\operatorname{Max}}-1\right)\right] \text {. }
$$

For $\theta: G_{\operatorname{Min}}, \theta_{2}^{\prime}$ shadowing occurs, and the expression for $\theta_{2}^{\prime}$ must be modified.

The 1 imiting value of $\theta$ for $\theta i$ shadowing $\left(\frac{d}{\rho}<\imath 2.0558\right)$ is shown in Figure B5.

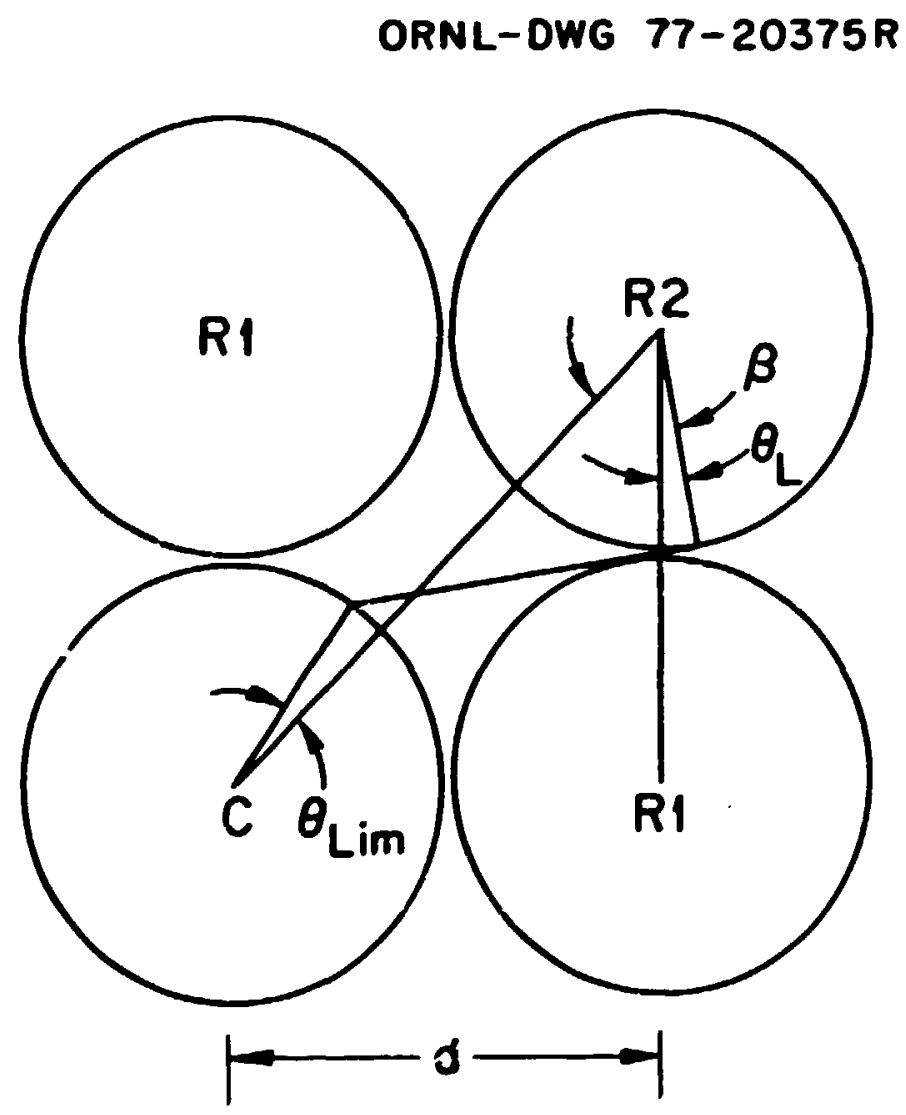

Figure B5. Limiting value of $\theta$ for $\theta_{1}$ shadowing. 
From Equation (A1). $\hat{A}_{L}=\cos ^{-1} \frac{20}{d}$,

$$
3=\frac{\bar{\pi}}{4}+\sigma_{L}=\overline{\bar{\pi}}+\cos ^{-1}\left(\frac{\bar{c}_{p}}{d}\right) .
$$

Considering ${ }^{A_{L}}$ im as a limiting angle as seen from $R 2$ at an angle 6 and at a distance $\sqrt{2} d$, Equation (A5) gives:

$$
\theta_{\text {Lim }}=\frac{\pi}{4}+\cos ^{-1}\left(\frac{20}{d}\right)-\cos ^{-1}\left\{\frac{\sqrt{2} d}{\rho} \cos \left[\frac{\pi}{4}+\cos ^{-1}\left(\frac{2 \rho}{d}\right)\right]-1\right\}
$$

for $\theta<e_{\text {Lim }}, \theta_{1}^{\prime}$ shadowing cccul's anc the expression for it must be modified.

Now we need the correct expressions for $\theta_{1}^{\prime}$ and $\theta_{:}^{\prime}$ to satisfy the shadowing conditions outl ined here. The case for $9_{2}^{\prime}$ is shown in Figure B6.

ORNL-OWG 77-20376

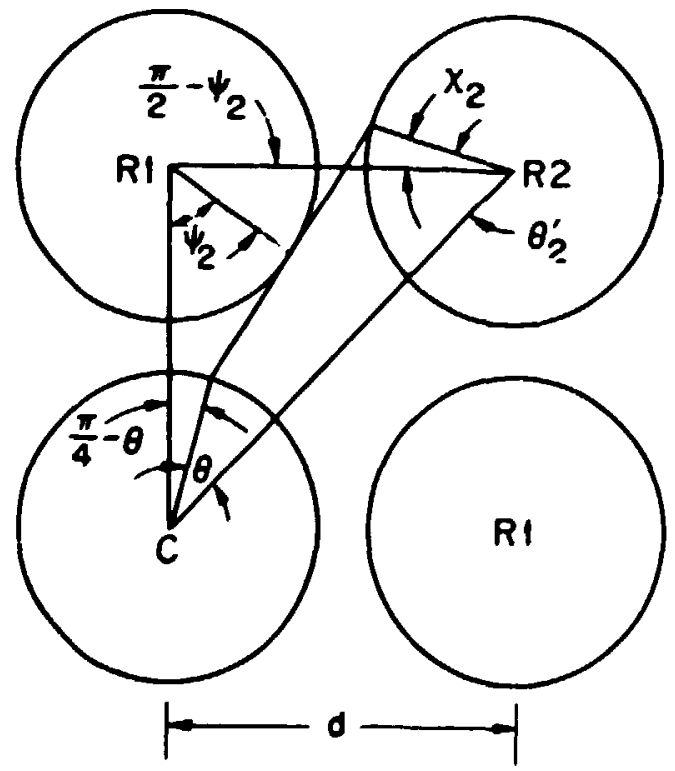

Figure B6. Limiting value, $\theta_{2}^{\prime}$. 
The angle $Y_{2}$ can be obtained from Equations (A2) and (A4), for an angle $\frac{\pi}{4}-\theta$, and:

$$
h_{2}=\sqrt{d^{2}-2 d \rho \cos \left(\frac{\pi}{4}-\theta\right)+\rho^{2}} \text {. }
$$

Thus we have:

$$
\psi_{2}=\cos ^{-1}\left(\frac{\rho}{h_{2}}\right)+\sin ^{-1}\left[\frac{\rho \sin \left(\frac{\pi}{4}-\theta\right)}{h_{2}}\right] .
$$

Likewise, $x_{2}$ can be obtained from Equation (A5) for an angle $\frac{\pi}{2}-\Psi_{2}$.

$$
x_{2}=\frac{\pi}{2}-\psi_{2}-\cos ^{-1}\left[\frac{d}{\rho} \cos \left(\frac{\pi}{2}-\psi_{2}\right)-1\right] \text {, }
$$

and:

$$
\theta_{2}^{\prime}=\frac{\pi}{4}+x_{2} \text {. }
$$

By using a similar path of reasoning for obtaining the expression for $\theta_{1}$, we can say that for this case the angle $\psi_{1}$ is obtained from Equations (A2) and (A4), for an angle $\frac{\pi}{4}+\theta$, and:

$$
\begin{aligned}
& h_{1}=\sqrt{d^{2}-2 d \rho \cos \left(\frac{\pi}{4}+\theta\right)+o^{2}} \\
& \psi_{1}=\cos ^{-1}\left(\frac{\rho}{h_{1}} \cdot\right)^{-i n} \sin ^{-1}\left[\frac{\rho \sin \left(\frac{\pi}{4}+\theta\right)}{h_{1}}\right] . \\
& x_{1}=\frac{\pi}{2}-\omega_{1}-\cos ^{-1}\left[\frac{d}{\rho} \cos \left(\frac{\pi}{2}-\psi_{1}\right)-1\right], \\
& \theta_{1}=\frac{\pi}{4}+x_{1} .
\end{aligned}
$$


35

To summarize the results, we have:

1. No R2 shadowing for $\frac{d}{5} \geq \frac{4}{\sqrt{2}}$.

2. For $\frac{d}{2}<\frac{4}{\sqrt{2}}$ and $\theta_{\operatorname{Min}}<\theta<\theta_{\operatorname{Max}}, \theta_{2}^{\prime}=\frac{\pi}{4}+\therefore$, and upper limit of $\epsilon$ is ${ }^{3} \operatorname{Max}$.

3. No R2 shadowing of $\theta_{1}^{\prime}$ for $\frac{d}{d} \geq \sim 2.0558$.

4. For $\frac{d}{6} \cdot 0,0558$ a: $\theta<\theta_{\lim }, \theta_{1}^{\prime}=\frac{\pi}{4}+x_{1}$. 


\section{APPENDIX C}

\section{CHANGES IN THE LIMITS OF INTEGRATION FOR}

TRIANGULAR PITCH ARRAYS OF CYL INDERS DUE TO SHADOWING EFFECTS

Consider a triangular array of cylinders of radius $\rho$ and having a pitch d. shown in Figure $\mathrm{Cl}$.

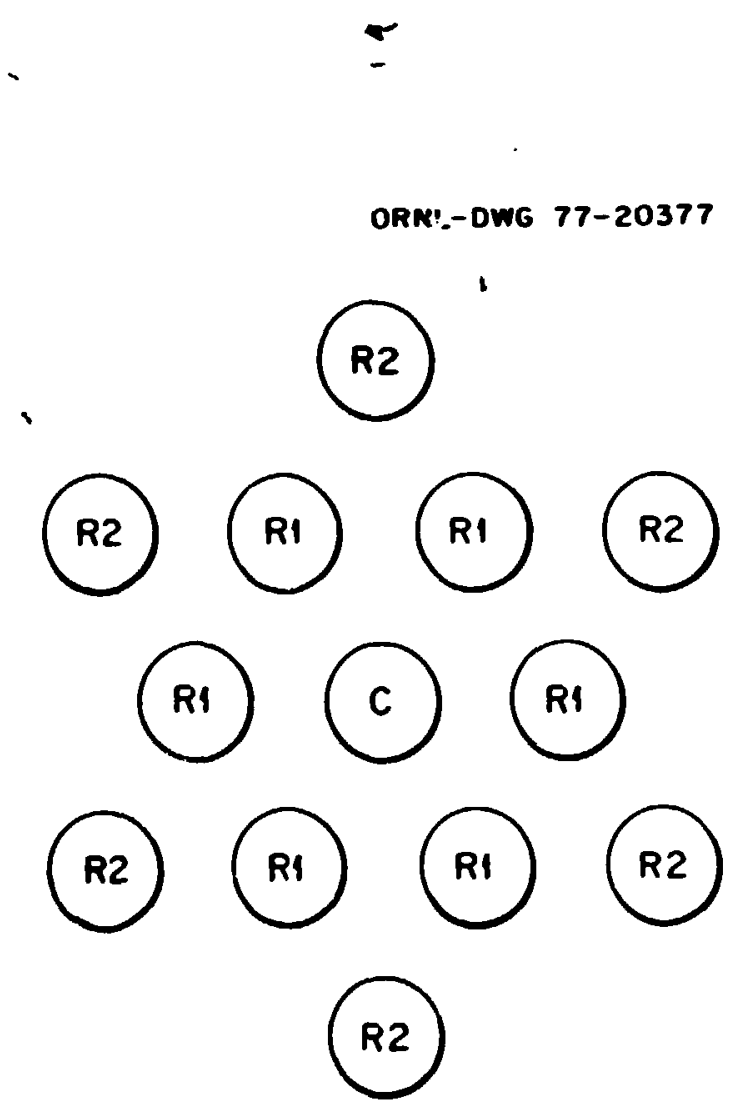

Figure $\mathrm{Cl}$. Triangular array of cylinders.

For the purpose of computing Dancoff factors, only the six nearest cylinders, designated $R 1$, and the six next nearest sylinders, designated $R 2$, are considered. For sufficiently close packed arrays, each cylinder $R I$ will be partially obscured by the Rl cylinders adjacent to it. Likewise. for spacing below some other 1imit, cylinders R2 will be partially obscured 


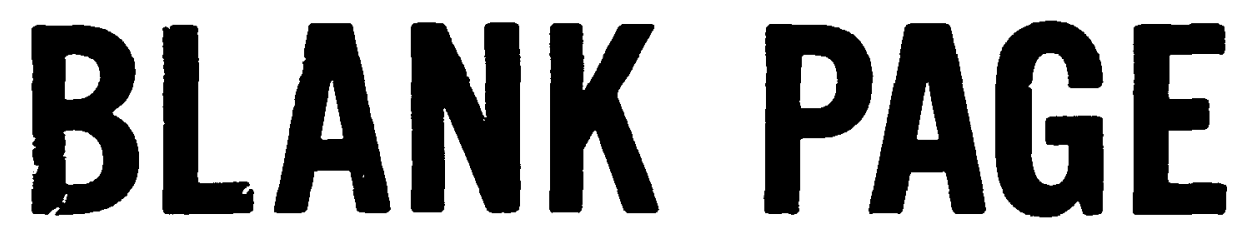


by cylinders RI. (In the extreme case, where the cyininers touch each other, the cylinders $R \bar{L}$ are comp?etely obscured.) Inder these conditions, the expressions for $\theta_{1}^{\prime}$ and $\theta_{2}^{\prime}$ must be modified. In addition, the top limit of the $\theta$ integration (above which the cylinder is totally obscured) must be reduced from $\frac{\pi}{2}$ to some $\theta_{\operatorname{Max}}<\frac{\pi}{2}$. The limiting spacing for shadowing of $\theta_{2}^{\prime}$ for $R 1$ cylinders is considered in Figure C2. No $C_{1}^{\prime}$ shadowing occurs for Rl cylinders.

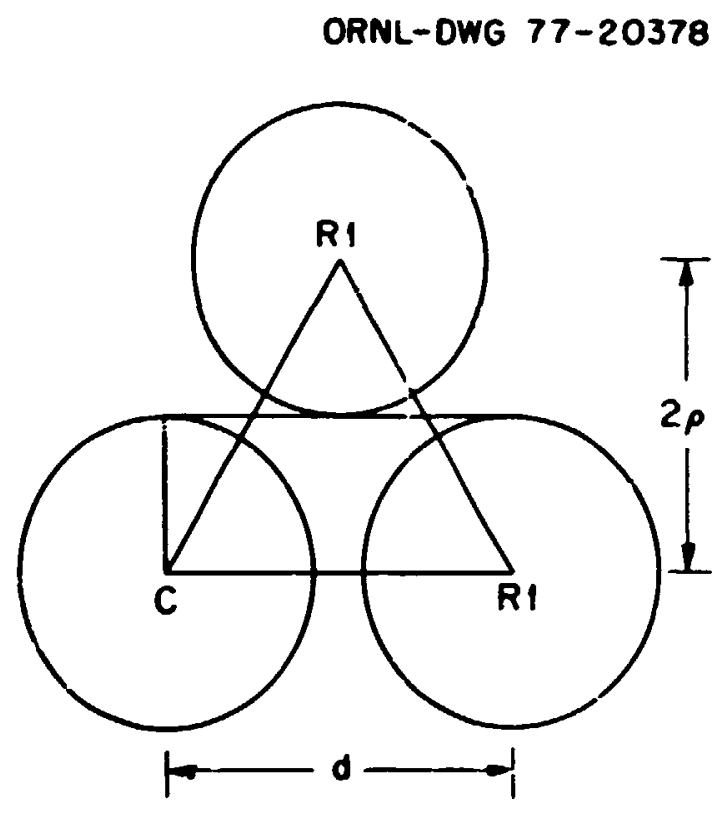

Figure C2. Limiting spacing for shadowing of $\partial_{2}^{\prime}$.

The altitude of the triangle shown is 20 . This altitude is also $\frac{\sqrt{3}}{2} \mathrm{C}$, thus:

$$
2 \rho=\frac{\sqrt{3}}{2} d, \frac{d}{n}=\frac{4}{\sqrt{3}} \text {. }
$$


For $\frac{d}{\bar{\rho}}<\frac{4}{\sqrt{\hat{3}}}$ and sufficientiy large values of $\theta$, the $\theta !$ expression must be modified.

The limiting valles of $\theta$ for $\theta_{2}^{\prime}$ shadowing $\left(\frac{d}{c}<\frac{4}{\sqrt{3}}\right)$ for $R I$ cylinders is shown in Figure $\mathrm{C} 3$.

ORNL- DWG 77-20379

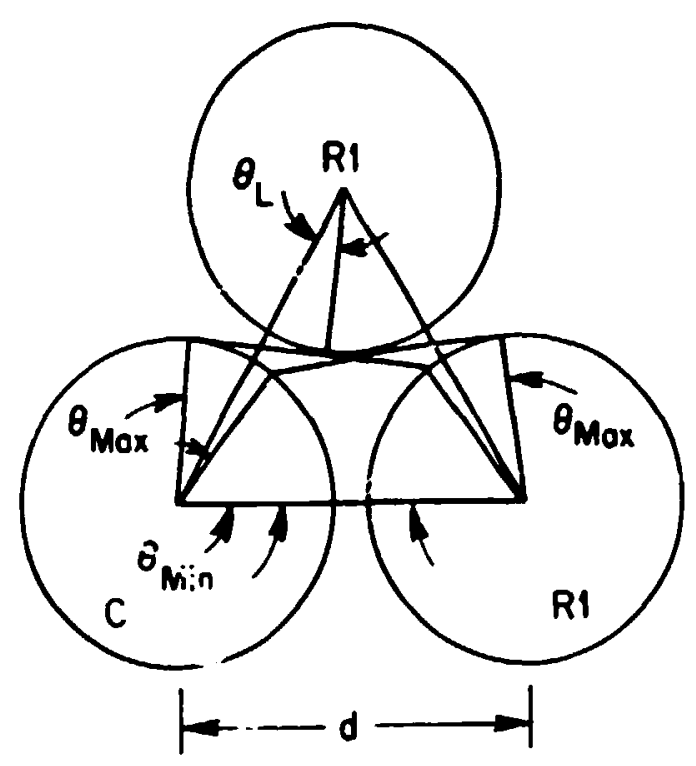

Figure $C 3$. Limiting values of 5 for $A_{2}^{\prime}$ shadowing.

Equation (Al) gives ${ }^{\prime} L=\cos ^{-1}\left(\frac{2 p}{d}\right)$.

$$
{ }^{\theta_{\operatorname{Max}}}=\frac{\pi}{3}+\theta_{L}=\frac{\pi}{3}+\cos ^{-1}\left(\frac{2_{B}}{d}\right) .
$$

For $\theta \geq \theta_{\text {Max }}$, the cylinder $R 1$ is totally obscured, and the upper limit of the $\theta$ integration must be reduced from $\frac{\pi}{2}$ to ${ }^{\theta_{\operatorname{Max}}}$. 
If we consider $\theta_{\text {Min }}$ as a limiting angie as seen from $R 1$ an angle "Max, Equation (A5) gives:

$$
{ }^{\theta}{ }_{\operatorname{Min}}=-\left[\theta_{\operatorname{Max}}-\cos ^{-1}\left(\frac{d}{0} \cos \theta_{\operatorname{Max}}-1\right)\right] \text {. }
$$

For $\theta>\theta_{\operatorname{Min}}, \theta_{2}^{\prime}$ shadowing occurs and the expression for $\theta_{2}^{\prime}$ must be modified. This expression may be obtained frnm figure C4.

ORNL-DWG 77-20380R

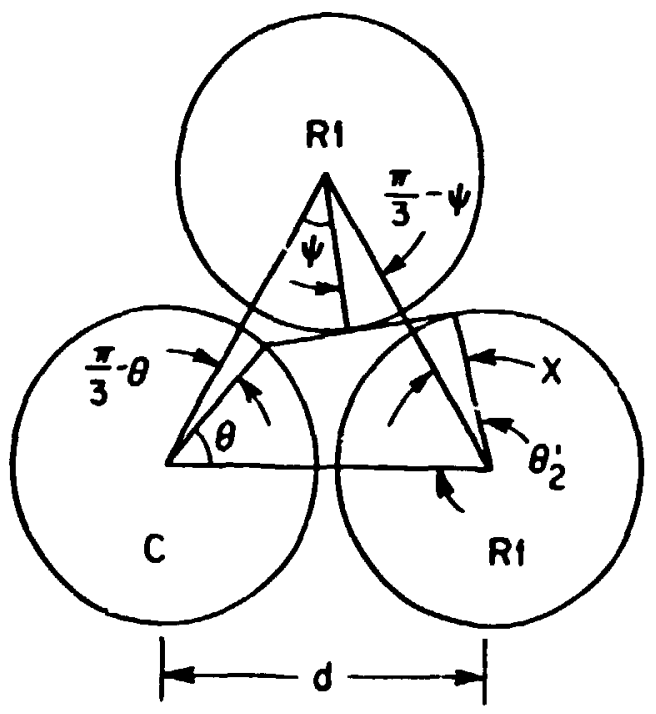

Fiqure C4. Limiting value, $\theta_{2}^{\prime}$.

The correct expression for $\theta_{2}^{\prime}$ for $R /$ which satisfies the condition may be obtained as follows. The angle $\Psi$ can be obtained from Equations (A2) and (A4), for an angle $\frac{\pi}{3}-\theta$ :

$$
\begin{aligned}
& h=\sqrt{d^{2}-2 d o \cos \left(\frac{\pi}{3}-\theta\right)+\rho^{2}}, \\
& \psi=\cos ^{-1}\left(\frac{\rho}{h}\right)+\sin ^{-1}\left[\frac{\rho \sin \left(\frac{\pi}{3}-\theta\right)}{h}\right] .
\end{aligned}
$$


Likewise, $x$ can be obtained from Equation (A5) for an angle $3-$ :

$$
x=\frac{\pi}{3}-\varphi-\cos ^{-1}\left[\frac{d}{\rho} \cos \left(\frac{\pi}{3}-\varphi\right)-1\right] \text {, }
$$

and

$$
\theta_{2}^{\prime}=\frac{\pi}{3}+x
$$

For rylinders R2, the 1 imiting spacing for $\theta_{2}^{\prime}$ shadowing is shown in Figure C5.

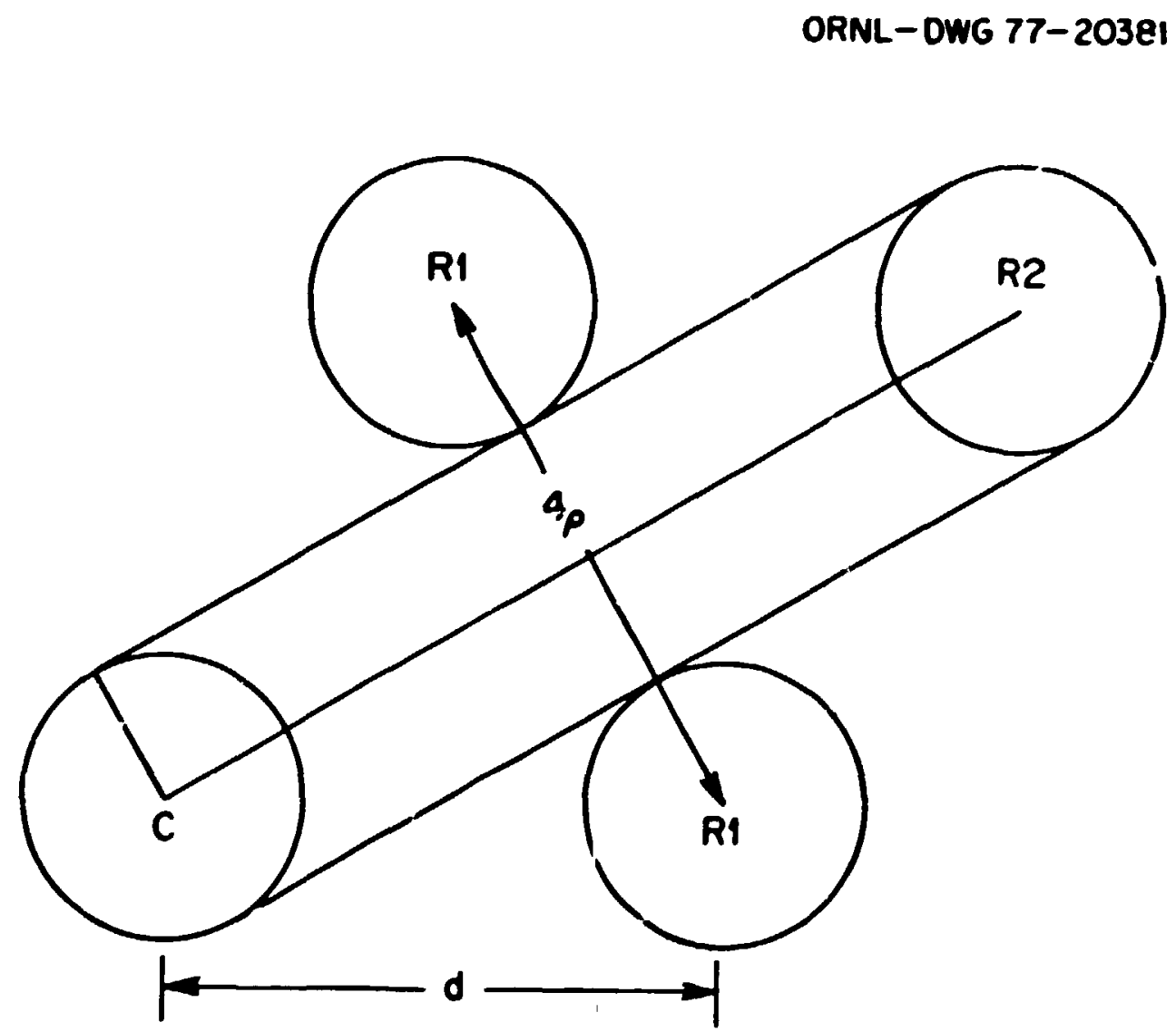

Figure C5. Limiting spacing for shadowing. 
From figure $\mathrm{C5}$, it can be seen that:

$$
\begin{aligned}
& d=4 \rho, \\
& \frac{d}{\rho}=4 .
\end{aligned}
$$

For $\frac{d}{\rho}<4$ and sufficiently large values of $\theta$, the $\theta_{2}^{\prime}$ expression must be modified.

Next, consider the limiting spacing for $\theta_{\mathrm{i}}$ shadowing of R2 cylinders, shown in Figure $\mathrm{C} 6$.

ORNL- OWG 77-20382

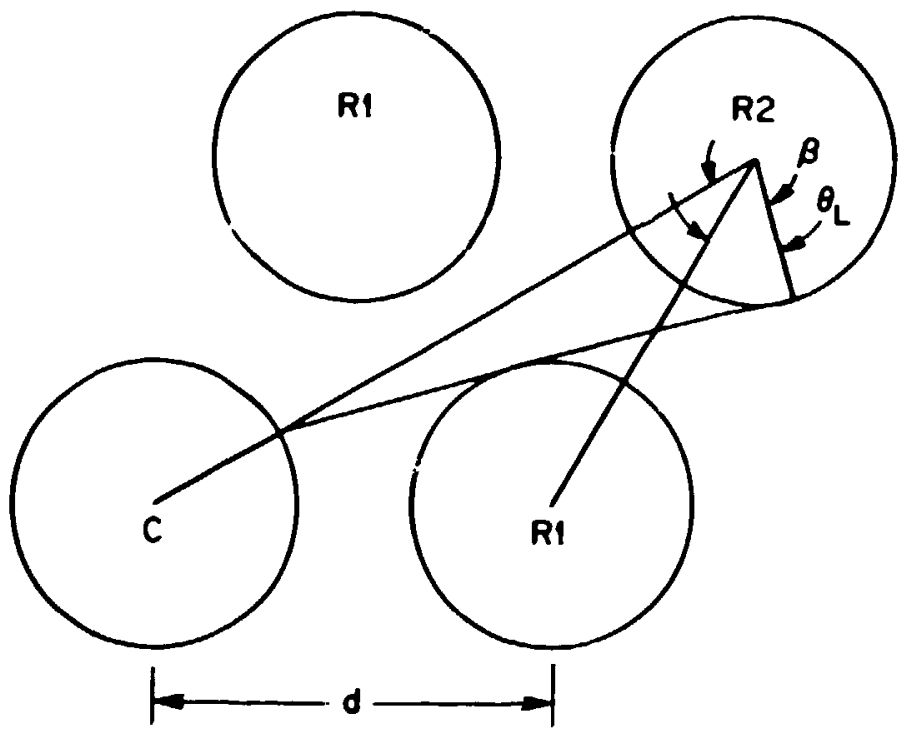

Figure C6. Limiting spacing for $\theta_{1}^{\prime}$ shadowing.

From Equation (A1), $\theta_{L}=\cos ^{-1}\left(\frac{2 \rho}{d}\right)$. 
Thus:

$$
B=\frac{\pi}{6}+\theta_{L}=\frac{\pi}{6}+\cos ^{-1}\left(\frac{2 \rho}{d}\right) .
$$

The value of $B$ can also be obtained from Equations (A2) and (A3), using an angle $\theta=0$ and a distance of $\sqrt{3} \mathrm{~d}$ :

$$
\begin{aligned}
& h=\sqrt{3 d^{2}-2 \sqrt{3} d \rho+\rho^{2}}, \\
& h=\sqrt{3} d-c, \\
& B=\cos ^{-1}\left(\frac{\rho}{\sqrt{3} d-\rho}\right) .
\end{aligned}
$$

For the limiting separation considered here, these two expressions for 8 must be equal.

$$
\frac{\pi}{6}+\cos ^{-1}\left(\frac{2 p}{d}\right)=\cos ^{-1}\left(\frac{p}{\sqrt{3} d-p}\right)
$$

Noting that:

$$
\sin ^{-1} \sqrt{1-\frac{40^{2}}{d^{2}}}=\cos ^{-1}\left(\frac{2}{d}\right)
$$

and using the expression for the cosine of the sum of two angles leads to:

$$
\begin{aligned}
& \frac{\sqrt{3}}{2}\left(\frac{2 p}{d}\right)-\frac{1}{2} \sqrt{1-\frac{4 p^{2}}{d^{2}}}=\frac{\rho}{\sqrt{3} d-0}, \\
& \frac{\sqrt{3}}{d / \rho}-\frac{1}{2} \sqrt{\left.1-\frac{4}{(d / \rho}\right)^{2}}=\frac{1}{\sqrt{3} d / 0-1} .
\end{aligned}
$$


This expression was solved for $\frac{d}{b}$ by an iterative procedure. Fron this:

$$
\frac{d}{\rho} \simeq 2.8388
$$

For values of $\frac{d}{o}$ less than this and for sufficiently small vaiues of $g$, the expression for $e_{i}$ must be modified.

The limiting values of $\theta$ for $\theta_{2}^{\prime}$ shadowing $\left(\frac{d}{\rho}<4\right)$ are show in Figure C7.

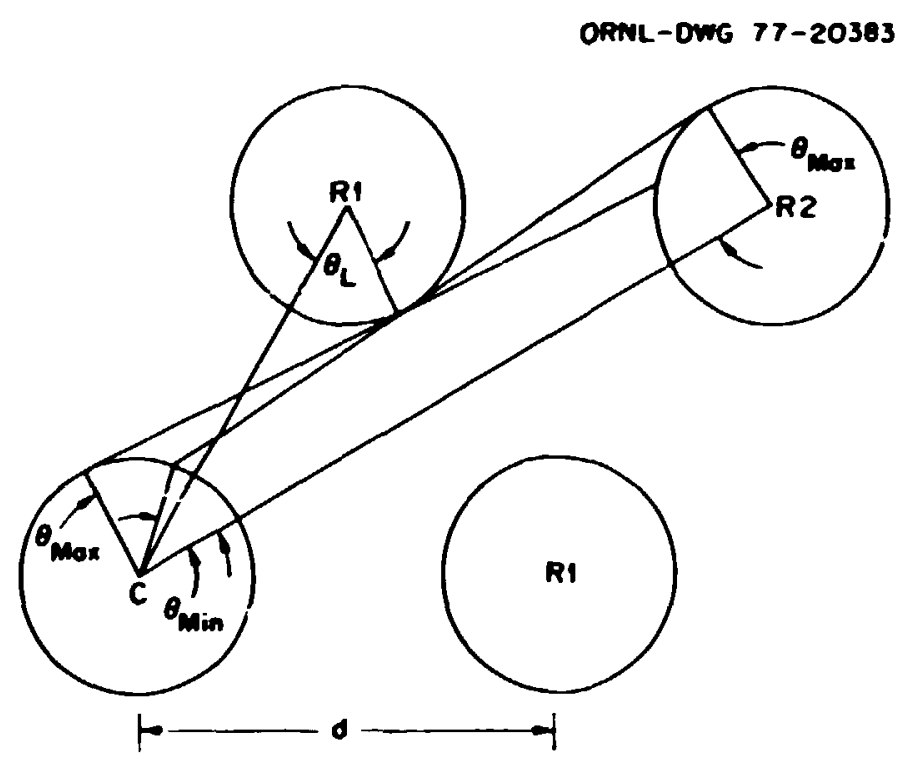

Figure $\mathrm{C} 7$. Limiting values of $\theta$ for $\theta_{2}^{\prime}$ shadowing.

From Equation (A1), $\theta_{L}=\cos ^{-1}\left(\frac{2 \rho}{d}\right)$.

$$
\theta_{\operatorname{Max}}=\frac{\pi}{6}+\theta_{L}=\frac{\pi}{6}+\cos ^{-1}\left(\frac{20}{d}\right)
$$


For values of $\exists \geq{ }^{A}$ Max , cylinder R2 is totally obscured. Thus, the upper limit of the $\subseteq$ integration is reduced from $\frac{\pi}{2}$ to ${ }^{\theta} \operatorname{Max}$.

Considering $\theta_{\text {Min }}$ as a limiting angle as seen from R2 at an angle ${ }^{9} \operatorname{Max}$ and a distance of $\sqrt{3} \mathrm{~d}$, Equation (A5) gives:

$$
\theta_{\operatorname{Min}}=-\left[\theta_{\operatorname{Max}}-\cos ^{-1}\left(\frac{\sqrt{3} d}{\tilde{\sigma}} \cos \theta_{\operatorname{Max}}-1\right)\right] \text {. }
$$

For $\theta>{ }^{M i n}$, $\theta_{2}^{\prime}$ shadowing requires the expression for $\theta_{2}^{\prime}$ to be modified.

The limiting value of $\theta$ for $\theta i$ shadowing of $R 2$ cylinders $\left(\frac{d}{0}<-2.8388\right)$ is shown in Figure $\mathrm{C} 8$.

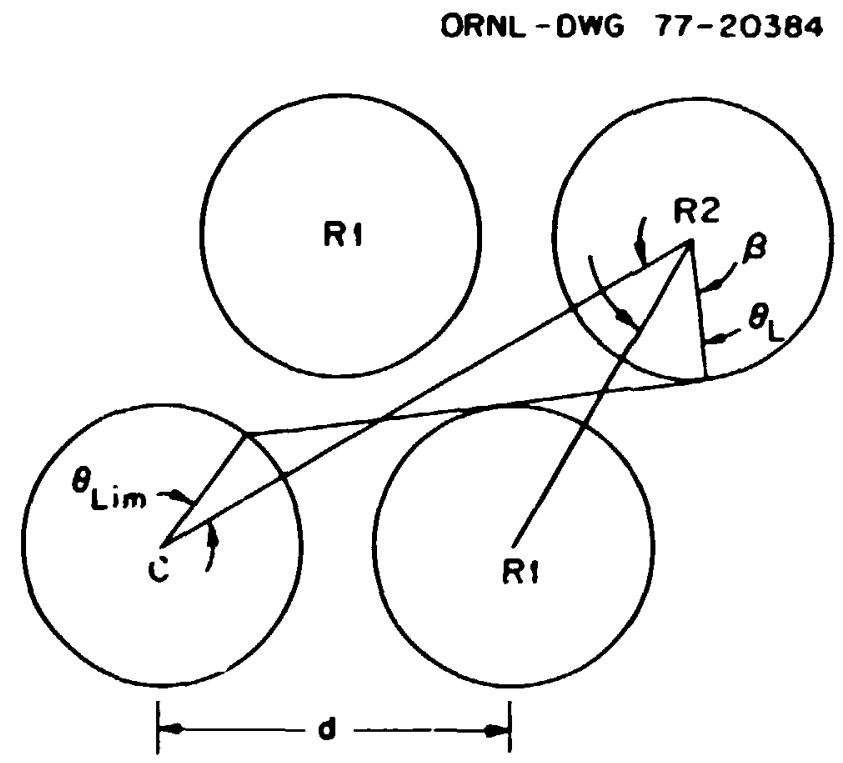

Figure C8. Limiting value of $A$ for $A_{1}^{\prime}$ shadowing.

From Equation (A1), ${ }^{A} L=\cos ^{-1}\left(\frac{2_{0}}{d}\right)$,

$$
B=\frac{\pi}{6}+\theta_{L}=\frac{\pi}{6}+\cos ^{-1}\left(\frac{2_{0}}{d}\right) .
$$


Considering $\theta_{\text {Lim }}$ as the liniting angle as seen from $R 2$ at an angle 2 and at a distance of $\sqrt{3} 1$, Equation (A5) gives:

$$
\theta_{\text {Lim }}=\frac{\pi}{6}+\cos ^{-1}\left(\frac{2 p}{d}\right)-\cos ^{-1}\left\{\frac{\sqrt{3}}{\rho} \frac{d}{\rho} \cos \left[\frac{\pi}{6}+\cos ^{-1}\left(\frac{2 \rho}{d}\right)\right]-1\right\}
$$

For $\theta<\theta_{\text {Lim }}, \theta_{i}$ shadowing requires that the expression for $\theta_{i}$ be modified.

Now we need the corrected expressions for $\theta_{1}^{\prime}$ and $\theta_{2}^{\prime}$ to satisfy the shadowing colditions described here. The case for $\theta_{2}^{\prime}$ is shown in Figure c9.

ORNL-OWG 77-20385

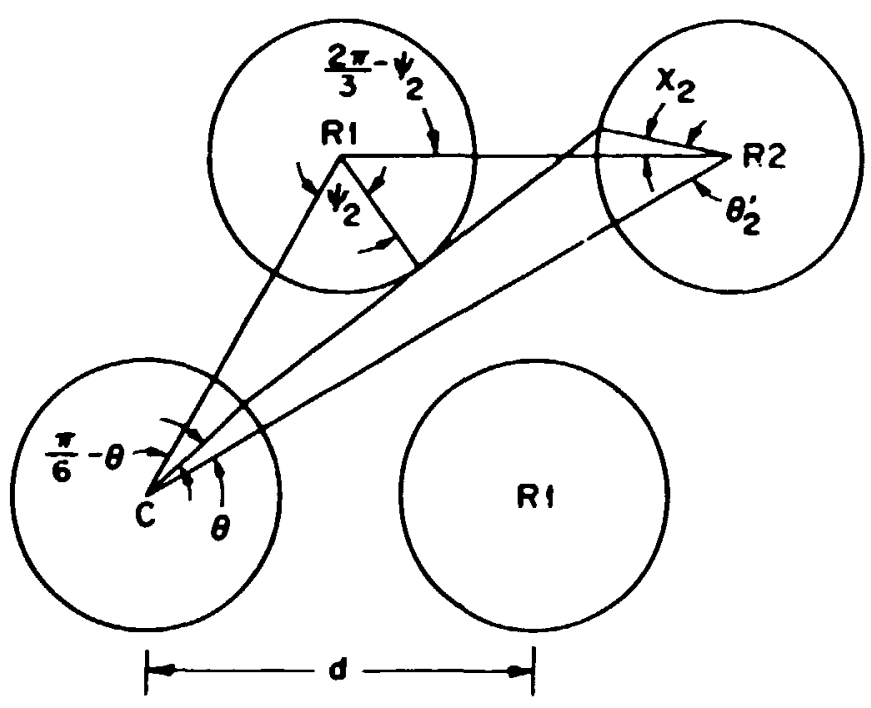

Figure C9. Limiting value, $\theta_{2}^{\prime}$.

The angle $\Psi_{2}$ can be obtained from Equations (A2) and (A4), for an angle $\frac{\pi}{6}-\theta:$

$$
h_{2}=\sqrt{d^{2}-2 d \rho \cos \left(\frac{\pi}{6}-\theta\right)+o^{2}} \text {. }
$$


Thus we have:

$$
r_{2}=\cos ^{-1}\left(\frac{\rho}{h_{2}}\right)+\sin ^{-1}\left[\frac{\sin \left(\frac{\pi}{6}-\rho\right)}{h_{2}}\right] \text {. }
$$

Similarly, $x_{2}$ Cä: te ubtained from Equation (A5) for an angle $\frac{\hat{z}^{-}}{3}-\vdots_{2}$ :

$$
x_{2}=\frac{2 \pi}{3}-i_{2}-\cos ^{-i}\left[\frac{d}{0} \cos \left(\frac{2 \pi}{3}-i_{i}\right)-1\right] \text {, }
$$

and:

$$
\theta_{2}^{\prime}=\frac{\pi}{6}+x_{2} \cdot
$$

By following a similar path of reasoniny, we can find the expression for $\theta_{1}^{\prime}$. The angle $y_{1}$ is obtained from Equations (A2) and (A.5) for an angle $\frac{\pi}{6}+6$ :

$$
\begin{aligned}
& h_{1}=\sqrt{d^{2}-2 d, \cos \left(\frac{\pi}{6}+\theta\right)+r_{1}^{2}}, \\
& \psi_{1}=\cos ^{-1}\left(\frac{\rho}{h_{1}}\right)+\sin ^{-1}\left[\frac{\sin \left(\frac{\pi}{6}+\theta_{0}\right)}{h_{2}}\right], \\
& x_{1}=\frac{2 \pi}{3}-\psi_{1}-\cos ^{-1}\left[\frac{d}{\rho} \cos \left(\frac{2 \pi}{3}-r_{1}\right)-1\right], \\
& \theta_{1}^{\prime}=\frac{\pi}{6}+x_{1} .
\end{aligned}
$$


To summarize the results, we have:

1. No RI shadowing for $\frac{d}{0} \geq \frac{4}{\sqrt{3}}$.

2. For $\frac{d}{0}<\frac{4}{\sqrt{3}}$ and $\theta_{\operatorname{Min}}<\theta<\theta_{\operatorname{Max}}(R 1), \theta_{2}^{\prime}=\frac{\pi}{3}+x$, and the upper limit of $\theta$ is $\theta_{\text {Max }}$.

3. No $R 2$ shadowing for $\frac{d}{\rho} \geq 4$.

4. For $\frac{d}{\rho}<4$ and $\theta_{\operatorname{Min}}<\theta<\theta_{\operatorname{Max}}(R 2), \theta_{2}^{\prime}=\frac{\pi}{6}+x_{2}$, and the upper limit of $\theta$ is $\theta_{\text {Max }}$.

5. No $R 2$ shadowing of $\theta_{i}$ for $\frac{d}{\rho} \geq \sim 2.8388$.

6. For $\frac{d}{\rho}<\sim 2.8388$ and $\theta<\theta_{\operatorname{Lim}}(R 2), \theta_{1}^{\prime}=\frac{\pi}{6}+x_{1}$. 


\section{APPENDIX D \\ THE EFFECT OF CLAU ON THE DANCOFF FAT:TOR}

IN CYLINDRICAL GEOMETRY

The expression for the Dancoff factor contains a term which takes intc account the effect of the moderating material separating the two cylinders. This is the Bickley function term, which is:

$$
\operatorname{ki}_{3}\left(\frac{|\overrightarrow{p-0}|}{x}\right),
$$

where $|\overrightarrow{\rho-\rho}|$ is the length of the path through the moderator and $\lambda$ is the mean free path of the moderator. As long as no clad is present, this term takes into account the attenuation in neutron flux at resonance energy due to the moderator. When clad is present, this term must be modified.

With clad surrounding the rods, the neutrons pass through a total clad path, $T_{C}$, and through a total moderator thickness, $|\overrightarrow{0-0}|-T_{C}$. If the mean free path of the neutrons in the clad is $\lambda_{c}$, the Bickley function term may be written:

$$
K_{j}\left(\frac{\left|\overrightarrow{0-0^{\prime}}\right|-T_{C}}{\lambda}+\frac{T_{C}}{\lambda_{C}}\right) .
$$

Thus, the total clad path must be determined for each neutron track considered.

The case for clad present is shown in Figure 01 . The thickness of the clad has been exaggerated to make the figure easier to interpret. 


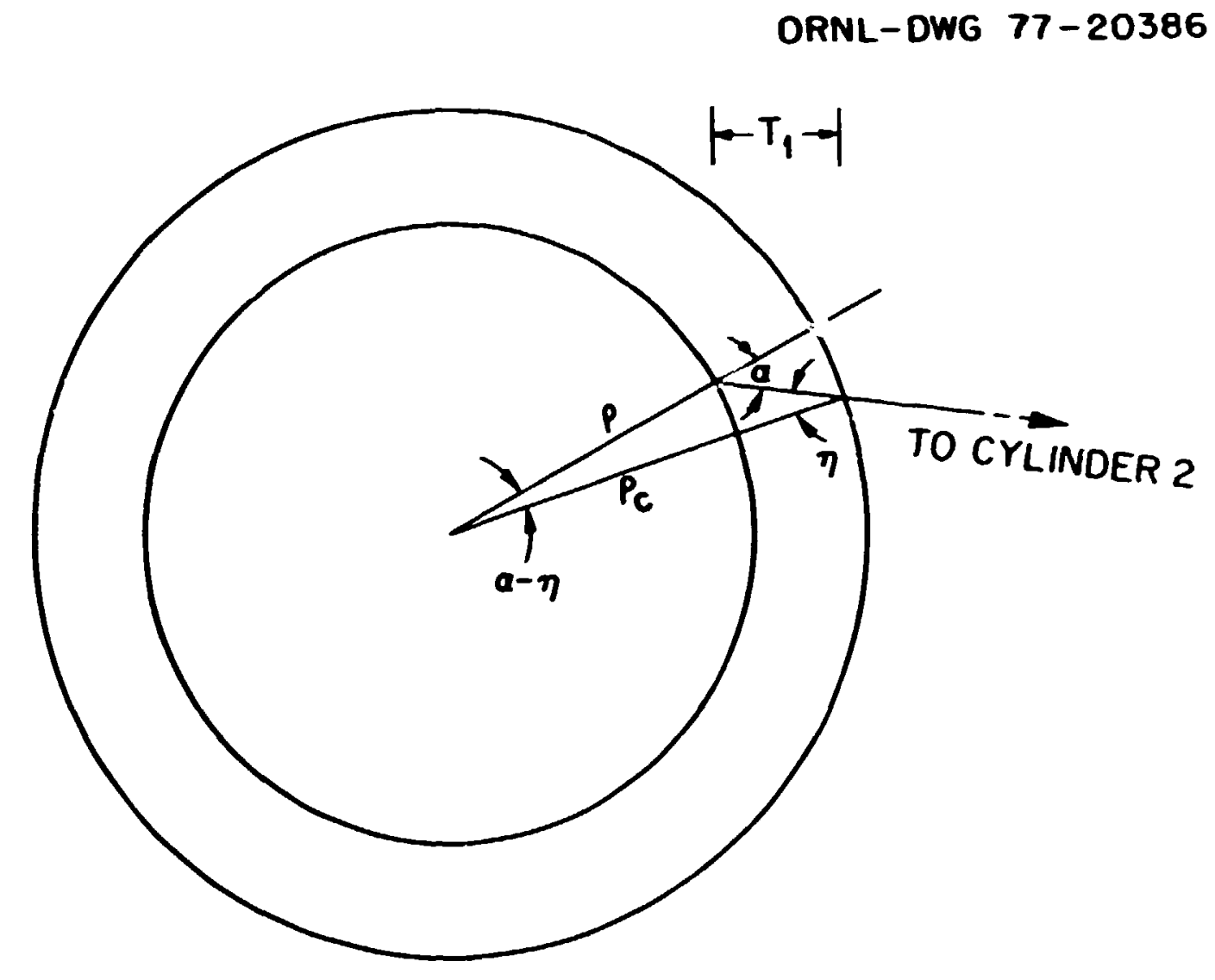

Figure D?. Cylinder with Clad. 
The quantities in Figrre $\mathrm{DI}$ are:

$$
\begin{aligned}
s= & \text { rod radius, } \\
i_{c}= & \text { clad outer radius, } \\
i= & \text { angle between the normal to the surface and the neutron path to } \\
& \text { the other cylinder. } \\
T_{1}= & \text { path length through clad of cylinder } 1 .
\end{aligned}
$$

From the law of sines, we have:

$$
\frac{\theta c}{\sin (\pi-a)}=\frac{\rho}{\sin \eta}=\frac{T_{1}}{\sin (a-\eta)} .
$$

Since $\sin (\pi-\alpha)=\sin \alpha$, this can be written:

$$
\frac{a_{c}}{\sin x}=\frac{\partial}{\sin n}=\frac{T_{i}}{\sin (x-n)}
$$

Dancoff and Ginsburg's expression for $x$ is:

$$
x=\cos ^{-1} \mid \frac{\mid d \cos \theta-0\left[1+\cos \left(\theta+\theta^{\prime}\right)\right] !}{|\rho-0|} !
$$

We also have:

$$
\begin{aligned}
& \eta=\sin ^{-1}\left(\frac{\partial}{\partial c} \sin x\right), \\
& T_{1}=\frac{n_{c} \sin (x-n)}{\sin x} .
\end{aligned}
$$


By a similar reasoning, the path length through the clad on cylinder 2 is:

$$
T_{2}=\frac{\rho c \sin \left(a^{\prime}-n^{\prime}\right)}{\sin a^{\prime}}
$$

where:

$$
a^{\prime}=\cos ^{-i}\left\{\frac{d \cos \theta^{\prime}-\rho\left[1+\cos \left(\theta+\theta^{\prime}\right)\right]}{\mid \overrightarrow{\rho-\partial^{\prime} \mid}}\right\} \text {, }
$$

and $\eta^{\prime}=\sin ^{-1}\left(\frac{\rho}{f_{C}} \sin a^{\prime}\right)$.

The total clad path is:

$$
T_{c}=T_{1}+T_{2}=\rho_{c}-\left[\frac{\sin (\alpha-\eta)}{\sin \alpha}+\frac{\sin \left(\alpha^{\prime}-\eta^{\prime}\right)}{\sin \alpha^{\prime}}\right] .
$$

When a gap is between the rod and the clad, the above expression must be modified. Sie have:

$$
T_{G_{1}}=\frac{P G \sin \left(a-\eta_{G}\right)}{\sin a}
$$

where ${ }^{D_{G}}=$ gap outer radius,

$$
\begin{aligned}
& T_{G_{1}}=\text { path length through the gap, and: } \\
& n_{G}=\sin ^{-1}\left(\frac{\rho}{\rho G} \sin \alpha\right) .
\end{aligned}
$$

Combining this with the expression for the gap in cylinder $2, T_{G_{2}}$, we obtain: 
53

$$
T_{G}=T_{G_{1}}+T_{G_{2}}={ }_{G}\left[\frac{\sin \left(\alpha-\cdot G_{2}\right)}{\sin \alpha}+\frac{\sin \left(\alpha^{\circ}-n_{2}^{\prime}\right)}{\sin \alpha^{\prime}}\right] .
$$

The modified expression for the total clad path, with a gap present, is:

$$
T_{C}=\rho_{C}\left[\frac{\sin \left(\alpha-r_{i}\right)}{\sin \alpha}+\frac{\sin \left(\alpha^{\prime}-n^{\prime}\right)}{\sin \alpha^{\prime}}\right]-T_{G} \text {, }
$$

and the corrected Buckley function term becomes

$$
K_{3}\left(\frac{\left|\rho-p^{\prime}\right|-T_{C}-T_{G}}{\lambda}+\frac{T_{i}}{\lambda_{C}}\right) \text {. }
$$


55

APPENDIX E

DERIVATION OF THE EXPRESSION FOR COS $\alpha$ FOR THE SPHERICAL DANCOFF FACTOR

Consider a plane passing through the centers of two spheres. A path from a point on sphere 1 to a point $\mathrm{P}^{\prime}$ on sphere 2 makes an angle $a$ with respect to the normal on sphere 1 . In Figure $E I$, the path and the point $P$ are above the plane described. Dark lines in the figure indicate lines above this plane. 


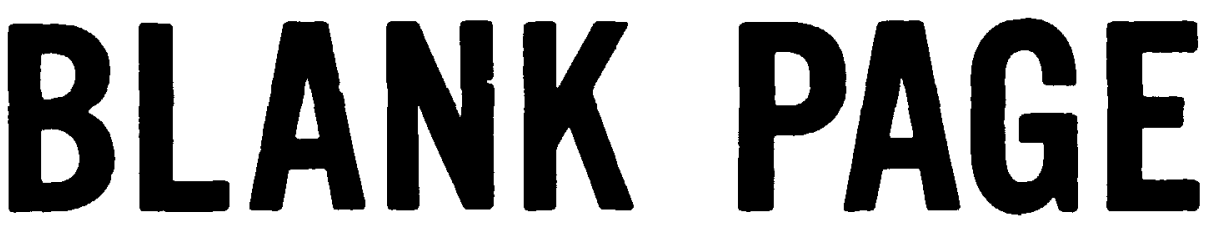


ORNL-OWG 77-2C S87R

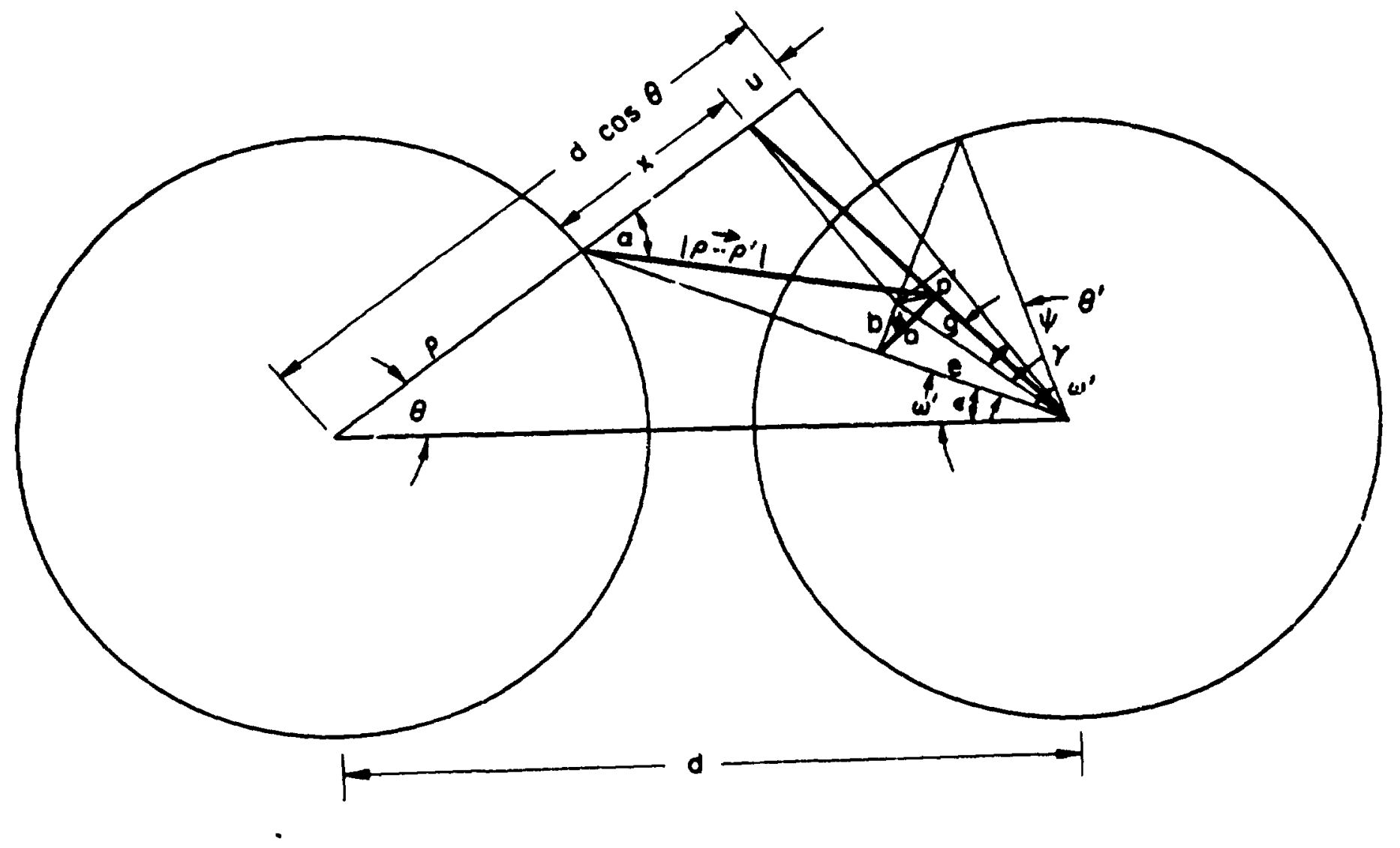

Fiqure El. Cos a for two spheres. 
From Figure $\mathrm{El}$, we have:

$$
\begin{aligned}
& a=\rho \sin \omega^{\prime}, \\
& \omega^{\circ}=\theta^{\circ}-\varepsilon .
\end{aligned}
$$

From Equation $(A 2)$, we have:

$$
\begin{aligned}
& \varepsilon=\sin ^{-1}\left(\frac{\rho \sin \theta}{\sqrt{d^{2}-2 d \rho \cos \theta+\rho^{2}}}\right) . \\
& b=a \cos \phi=\rho \sin \omega^{\prime} \cos \phi . \\
& e=\rho \cos \omega^{\prime} .
\end{aligned}
$$

Noting that:

$$
\varepsilon+\gamma+\psi=\frac{\pi}{2}-\theta,
$$

we have:

$$
\begin{aligned}
& \psi=\frac{\pi}{2}-\theta-\varepsilon-\gamma . \\
& u=g \sin \psi=g \sin \left[\frac{\pi}{2}-\theta-\varepsilon-\gamma\right] .
\end{aligned}
$$

Therefore:

$$
\begin{aligned}
& u=g \cos [\theta+\varepsilon+\gamma] . \\
& \gamma=\sin ^{-1}\left(\frac{b}{g}\right)=\cos ^{-1}\left(\frac{e}{g}\right) .
\end{aligned}
$$

If we take the expression for the cosine of the sum of two angles, where $\theta+\varepsilon$ is taken as one angle and $\gamma$ is taken as the other, we get: 


$$
\begin{aligned}
& u=g \cos (\theta+\varepsilon) \cos \gamma-g \sin (\theta+\varepsilon) \sin Y, \\
& u=g \cos (\theta+\varepsilon)\left(\frac{\rho \cos \omega^{\circ}}{g}\right)-g \sin (\theta+\varepsilon)\left(\frac{\partial \sin \dot{\prime}^{\prime} \cos \theta}{g}\right), \\
& u=\rho \cos (\theta+\varepsilon) \cos \omega^{\prime}-\partial \sin (\theta+\varepsilon) \sin \omega^{\prime} \cos \theta .
\end{aligned}
$$

The expression for $\alpha$ is:

$$
\begin{aligned}
& \cos a=\frac{x}{\left|\rho_{-\rho^{\prime}}\right|}, \\
& x=d \cos \theta-\rho-u, \text { and, since }\lrcorner^{\prime}=\theta^{\prime}-\varepsilon, \\
& x=d \cos \theta-\rho-\rho \cos (\theta+\varepsilon) \cos \left(\theta^{\prime}-\varepsilon\right) \\
& \quad+\rho \sin (\theta+\varepsilon) \sin \left(\theta^{\prime}-\varepsilon\right) \cos \phi .
\end{aligned}
$$

When we evaluate the expression used to obtain the Dancoff factor, it must be integrated over ali values of the angles. For small values of 0 and $\theta^{\prime}$, the integral covers the limits of $\theta$ from $C$ to $2 \pi$. However, for some limiting larger values of $\theta$ or $\theta^{\prime}$, the lower part of the circular path cannot be "seen" through moderator only, and the $p$ integration must be $\supset$ f the form:

$$
2 \int_{H}^{\phi_{M}} d \phi
$$

where $\phi_{M}$ is the limiting value of for the path passing through moderator only. This condition is met when:

$$
\Phi_{M} \leq \pi \text {, and } \cos a=0 \text {. }
$$


This second condition implies that the path is tangent to sphere 1, and any further increase in: is not possible.

From Equation (E7), we have:

$$
\begin{aligned}
& x=d \cos \hat{y}-c-\partial \cos (\hat{y}+\varepsilon) \cos (\hat{y}-\varepsilon) \\
& +2 \sin (\hat{\theta}+\varepsilon) \cos \left(\xi^{\prime}-\varepsilon\right) \cos : \text {. } \\
& \text { For } \cos x=0, x=0 \text {, and } \hat{t}=s_{M} \text { : } \\
& A_{M}=\cos ^{-1}\left[\frac{\rho+\rho \cos (\hat{\theta}+\varepsilon) \cos \left(\partial^{\prime}-\varepsilon\right)-d \cos \theta}{\partial \sin (\theta+\varepsilon) \sin \left(\theta^{\prime}-\varepsilon\right)}\right] \text {. }
\end{aligned}
$$

The expression for $x$ [Equation (E7)] is the only term in the spherical Dancoff equation which depends on the value of $\therefore$. Therefore, the * integration can be carried out as follows:

$$
\begin{aligned}
& \int_{0}^{\theta} d s[d \cos \theta-\theta-\theta \cos (\theta+\varepsilon) \cos (\therefore-\rho) \\
& +\therefore \sin (\theta+\varepsilon) \sin (\theta-\varepsilon) \cos \Leftrightarrow]
\end{aligned}
$$

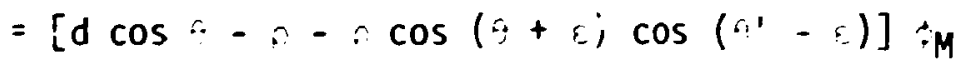

$$
\begin{aligned}
& +\theta \sin (\sigma+\varepsilon) \sin (\theta-\varepsilon) \sin M \text {. }
\end{aligned}
$$




\section{APPENDIX $F$ \\ METHOD FOR CALCULATING VALUES OF THE $E_{3}(x)$ FUNCTION}

The expression used to calculate the $E_{3}(x)$ function was obtained from AMS 55, ${ }^{7}$ Equation (5.1.12).

$$
\begin{aligned}
& E_{n}(x)=\frac{(-x)^{n-1}}{(n-1) !}[-\ln x+\Psi(n)]-\sum_{\substack{m=0 \\
m \neq n-1}}^{\infty} \frac{(-x)^{m}}{(m-n+1) m !}, \\
& \Psi(n)=-\gamma+\sum_{\substack{n-1 \\
m=1}}^{n} \frac{1}{m} .
\end{aligned}
$$

In the second expression, $\gamma$ is Euler's constant. For the case of interest here, $n=3$, and:

$$
\begin{aligned}
& E_{3}(x)=\frac{(-x)^{2}}{2 !}[-\ln x+\Psi(3)]-\sum_{\substack{m=0 \\
m \neq 2}}^{\infty} \frac{(-x)^{m}}{(m-2)^{m} !}, \\
& \Psi(3)=-\gamma+\sum_{m=1}^{2} \frac{1}{m}=-\gamma+\frac{3}{2} .
\end{aligned}
$$

This infinite series coniverges rapidly over the range of values of $x$ usually encountered in calculating the Dancoff factors of slabs. The value of $\gamma$ is given to 24 significant figures in Table 1.1 of AMS 55. Equation (FI) will not yield the function value for $x=0$. However, from AMS 55, Equation (5.1.23),

$$
\begin{aligned}
& E_{n}(0)=\frac{1}{n-1}(n<1), \\
& E_{3}(0)=\frac{1}{2} .
\end{aligned}
$$

